TRANSACTIONS OF THE

AMERICAN MATHEMATICAL SOCIETY

Volume 35, Number 7, Pages 2773-2804

S 0002-9947(01)02700-3

Article electronically published on March 15, 2001

\title{
$S L_{n}$-CHARACTER VARIETIES AS SPACES OF GRAPHS
}

\author{
ADAM S. SIKORA
}

\begin{abstract}
An $S L_{n}$-character of a group $G$ is the trace of an $S L_{n}$-representation of $G$. We show that all algebraic relations between $S L_{n}$-characters of $G$ can be visualized as relations between graphs (resembling Feynman diagrams) in any topological space $X$, with $\pi_{1}(X)=G$. We also show that all such relations are implied by a single local relation between graphs. In this way, we provide a topological approach to the study of $S L_{n}$-representations of groups.

The motivation for this paper was our work with J. Przytycki on invariants of links in 3-manifolds which are based on the Kauffman bracket skein relation. These invariants lead to a notion of a skein module of $M$ which, by a theorem of Bullock, Przytycki, and the author, is a deformation of the $S L_{2}$-character variety of $\pi_{1}(M)$. This paper provides a generalization of this result to all $S L_{n}$-character varieties.
\end{abstract}

\section{INTRODUCTION}

In this paper we introduce a new method in the study of representations of groups into affine algebraic groups. Although we consider only $S L_{n}$-representations, the results of this paper can be generalized to other affine algebraic groups; see [Si].

For any group $G$ and any commutative ring $R$ with 1 there is a commutative $R$-algebra $\operatorname{Rep}_{n}^{R}(G)$ and the universal $S L_{n}$-representation

$$
j_{G, n}: G \rightarrow S L_{n}\left(\operatorname{Rep}_{n}^{R}(G)\right)
$$

such that any representation of $G$ into $S L_{n}(A)$, where $A$ is an $R$-algebra, factors through $j_{G, n}$ in a unique way. This universal property uniquely determines $\operatorname{Rep}_{n}^{R}(G)$ and $j_{G, n}$ up to an isomorphism.

$G L_{n}(R)$ acts on $\operatorname{Rep}_{n}^{R}(G)$ (see Section 2), and the subring of $\operatorname{Rep}_{n}^{R}(G)$ composed of the elements fixed by the action, $\operatorname{Rep}_{n}^{R}(G)^{G L_{n}(R)}$, is called the the universal $S L_{n}$-character ring of $G$. This ring contains essential information about $S L_{n}$-representations of $G$. In particular, if $R$ is an algebraically closed field of characteristic 0 , then there are natural bijections between the following three sets:

- the set of all $R$-algebra homomorphisms $\operatorname{Rep}_{n}^{R}(G)^{G L_{n}(R)} \rightarrow R$,

- the set of all semisimple $S L_{n}(R)$-representations of $G$ up to conjugation, and

- the set of $S L_{n}(R)$-characters of $G$.

It is convenient to think about $\operatorname{Rep}_{n}^{R}(G)^{G L_{n}(R)}$ as the coordinate ring of a scheme, $\mathfrak{X}_{n}(G)=\operatorname{Spec}\left(\operatorname{Rep}_{n}^{R}(G)^{G L_{n}(R)}\right)$, called the $S L_{n}$-character variety of $G$.

Received by the editors May 18, 1999 and, in revised form, August 9, 2000.

1991 Mathematics Subject Classification. Primary 20C15, 57M27.

Key words and phrases. Character, character variety, skein module.

Partially supported by NSF grant DMS93-22675. 
As explained in Section 6, the algebra $\operatorname{Rep}_{n}^{R}(G)^{G L_{n}(R)}$ encodes all algebraic relations between the $S L_{n}$-characters of $G$. Unfortunately, it is very difficult to give a finite presentation of $\operatorname{Rep}_{n}^{R}(G)^{G L_{n}(R)}$ and, hence, to describe $\mathfrak{X}_{n}(G)$, even for groups $G$ with relatively simple presentations.

In this paper, we present a topological approach to the study of $S L_{n}$-character varieties. We prove that $R\left[\mathfrak{X}_{n}(G)\right]=\operatorname{Rep}_{n}^{R}(G)^{G L_{n}(R)}$ is spanned by a special class of graphs (resembling Feynman diagrams) in $X$, where $X$ is any topological space with $\pi_{1}(X)=G$; see Theorem [3.7 Moreover, all relations between the elements of this spanning set are induced by specific local relations between the graphs, called skein relations.

We postpone a detailed study of applications of our graphical calculus to the theory of $S L_{n}$-representations of groups to future papers. In this paper, we content ourself with an example, in which we apply our method to a study of $S L_{3^{-}}$representations of the free group on two generators. In this algebraically non-trivial example a huge reduction of computational difficulties can be achieved by the application of our geometric method.

This work is related to several areas of mathematics and physics:

Knot theory (and skein modules). Skein relations between links were used to define the famous polynomial invariants of links, like the Conway, Jones, and Homfly polynomials, [Co, Jo, FYHLMO, P-T, Ka]. In this paper we apply skein relations to the representations of groups.

The motivation for this work was our earlier work on skein modules, [PS-2]. The main theorem of this paper generalizes the Bullock-Przytycki-Sikora theorem relating the Kauffman bracket skein module of a manifold $M$ to the $S L_{2}(\mathbb{C})$-character variety of $\pi_{1}(M)$; see [B-2, $\mathrm{PS}-2$.

Quantum invariants of 3-manifolds. We hope that this work will help understand the connections between quantum invariants of 3-manifolds and representations of their fundamental groups. It follows from the work of Yokota $\mathrm{Yo}$ that for any 3-manifold $M$, the $S U_{n}$-quantum invariants of $M$ can be defined by using our graphs considered up to relations which are $q$-deformations of our skein relations.

Spin networks and gauge theory. The graphs considered in this paper have an interpretation as spin networks; see [Si]. They are also very similar to graphs used by physicists in non-abelian gauge theory (QCD); see [CV].

Number theory. After a preliminary version of this paper was made available, M. Kapranov pointed out to us that our work is related to the work of Wiles and others on "pseudo-representations." In his work (related to Fermat's Last Theorem), Wiles gave necessary and sufficient conditions under which a complex-valued function on $G$ is a $G L_{2}(\mathbb{C})$-character of $G$. His ideas were developed further and generalized to all $G L_{n}$-characters by Taylor, $\mathrm{Ta}$. See also $\mathrm{Ny}, \mathrm{Ro}$. These results provide a description of the coordinate ring of $G L_{n}$-character varieties quotiented by nilpotent elements. Our results are similar in spirit, but they are concerned with $S L_{n}$-representations and they are stronger, since they describe $R\left[\mathfrak{X}_{n}(G)\right]$ (i.e. $\left.\operatorname{Rep}_{n}^{R}(G)^{G L_{n}(R)}\right)$ exactly (with possible nilpotent elements).

The plan of this paper is as follows. In Section 2 we introduce some basic notions and facts concerning representations of groups. In Section 3 we define the algebra $\mathbb{A}_{n}(X)$ in terms of graphs in $X$ and formulate (Theorems 3.6 and 3.7) the main results of the paper asserting that $\mathbb{A}_{n}(X)$ is isomorphic to $\operatorname{Rep} p_{n}^{R}(G)^{G L_{n}(R)}$, where 
$G=\pi_{1}(X)$. The proof requires introducing another algebra, $\mathbb{A}_{n}\left(X, x_{0}\right)$, associated with any pointed topological space $\left(X, x_{0}\right)$. The algebra $\mathbb{A}_{n}\left(X, x_{0}\right)$ is an interesting object by itself, and for $n=2$ it already appeared in the theory of skein modules as a relative skein algebra. Sections 4 and 5 are devoted to the proof of the results of Section 3. In the final section we consider trace identities and use our results to describe the $S L_{3}$-character variety of the free group on two generators.

I would like to thank C. Frohman, B. Goldman, and J. H. Przytycki for many helpful conversations. C. Frohman explained some ideas to me which I subsequently used in this paper. Additionally, I would like to thank C. Frohman and W. Goldman for helping me make this paper more "reader-friendly".

\section{BACKGROUND FROM REPRESENTATION THEORY}

In this section we introduce the basic elements of the theory of $S L_{n}$-representations of groups. We follow the approach of Brumfiel and Hilden, ([B-H] , Chapter 8), which although formally restricted to $S L_{2}$-representations, has a straightforward generalization to $S L_{n}$-representations for any $n$. Compare also [L-M], [Pro-2].

Let $G$ be a group and let $R$ be a commutative ring with unity. There is a commutative $R$-algebra $\operatorname{Rep}_{n}^{R}(G)$, called the universal representation algebra, and the universal representation

$$
j_{G, n}: G \rightarrow S L_{n}\left(\operatorname{Rep}_{n}^{R}(G)\right)
$$

with the following property: For any commutative $R$-algebra $A$ and any representation $\rho: G \rightarrow S L_{n}(A)$ there is a unique homomorphism of $R$-algebras $h_{\rho}: \operatorname{Rep}_{n}^{R}(G) \rightarrow A$ which induces a homomorphism of groups

$$
S L_{n}\left(h_{\rho}\right): S L_{n}\left(\operatorname{Rep}_{n}^{R}(G)\right) \rightarrow S L_{n}(A)
$$

such that the following diagram commutes:

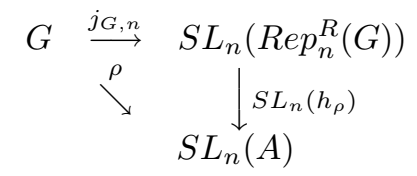

This universal property uniquely determines $\operatorname{Rep}_{n}^{R}(G)$ up to an isomorphism of $R$-algebras.

The universal representation algebra of $G$ may also be constructed explicitly in the following way. Let $\left\langle g_{i}, i \in I \mid r_{j}, j \in J\right\rangle$ be a presentation of $G$ such that all relations $r_{j}$ are monomials in non-negative powers of generators, $g_{i}$. Such a presentation exists for every group $G$. Since we work with groups which are not necessarily finitely presented, $I$ and $J$ may be infinite. Let $P_{n}(I)$ be the ring of polynomials over $R$ in variables $x_{j k}^{i}$, where $i \in I$ and $j, k \in\{1,2, \ldots, n\}$. Let $A_{i}$, for $i \in I$, be the matrix $\left(x_{j k}^{i}\right) \in M_{n}\left(P_{n}(I)\right)$. For any word $r_{j}=g_{i_{1}}^{n_{1}} g_{i_{2}}^{n_{2}} \ldots g_{i_{k}}^{n_{k}}$ consider the corresponding matrix $M_{j}=A_{i_{1}}^{n_{1}} A_{i_{2}}^{n_{2}} \ldots A_{i_{k}}^{n_{k}} \in M_{n}\left(P_{n}(I)\right)$. Let $\mathcal{I}$ be the two-sided ideal in $P_{n}(I)$ generated by polynomials $\operatorname{Det}\left(A_{i}\right)-1$, for $i \in I$, and by all entries of matrices $M_{j}-I d$, for $j \in J$, where $I d$ is the identity matrix. We denote the quotient $P_{n}(I) / \mathcal{I}$ by $\operatorname{Rep}_{n}^{R}(G)$ and the quotient map $P_{n}(I) \rightarrow \operatorname{Rep}_{n}^{R}(G)$ by $\eta$. Let $\bar{x}_{j k}^{i}=\eta\left(x_{j k}^{i}\right)$ and $\bar{A}_{i}=\left(\bar{x}_{j k}^{i}\right) \in M_{n}\left(\operatorname{Rep}_{n}^{R}(G)\right)$.

Note that we divided $P_{n}(I)$ by all relations necessary for the existence of a representation

$$
j_{G, n}: G \rightarrow S L_{n}\left(\operatorname{Rep}_{n}^{R}(G)\right)
$$


such that $j_{G, n}\left(g_{i}\right)=\bar{A}_{i}$. The algebra $\operatorname{Rep}_{n}^{R}(G)$ and the representation $j_{G, n}: G \rightarrow$ $S L_{n}\left(\operatorname{Rep}_{n}^{R}(G)\right)$ are exactly the universal representation algebra and the universal $S L_{n}$-representation of $G$.

Let $A \in G L_{n}(R)$. By the definition of the universal representation ring, $\operatorname{Rep} p_{n}^{R}(G)$, there is a unique homomorphism $f_{A}: \operatorname{Rep}_{n}^{R}(G) \rightarrow \operatorname{Rep}_{n}^{R}(G)$ such that the following diagram commutes:

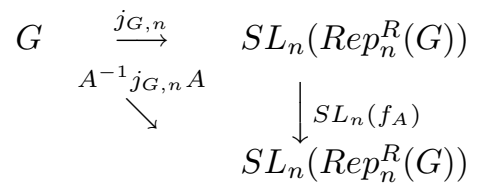

One easily observes that $f_{A}$ is an automorphism of $\operatorname{Rep}_{n}^{R}(G)$ and that the assignment $A \rightarrow f_{A}$ defines a left action of $G L_{n}(R)$ on $\operatorname{Rep}_{n}^{R}(G)$. We denote this action by $A *$, i.e. $f_{A}(r)=A * r$, for any $r \in \operatorname{Rep}_{n}^{R}(G)$ and $A \in G L_{n}(R)$. We call the ring $\operatorname{Rep}_{n}^{R}(G)^{G L_{n}(R)}$ consisting of elements of $\operatorname{Rep}_{n}^{R}(G)$ fixed by the action of $G L_{n}(R)$ the universal character ring of $G$. This term indicates a connection between the ring $\operatorname{Rep}_{n}^{R}(G)^{G L_{n}(R)}$ and $S L_{n}$-characters of $G$, i.e. traces of $S L_{n}$-representations of $G$. In the simplest case, when $R$ is an algebraically closed field of characteristic 0 , and $G$ is finitely generated, this connection can be described as follows. Every representation $\rho: G \rightarrow S L_{n}(R)$ induces a homomorphism $h_{\rho}: \operatorname{Rep}_{n}^{R}(G) \rightarrow R$, whose restriction to $\operatorname{Rep}_{n}^{R}(G)^{G L_{n}(R)}$ we denote by $h_{\rho}^{\prime}$. The next proposition follows from geometric invariant theory and from the results of $[\mathrm{L}-\mathrm{M}]$.

Proposition 2.1. Under the above assumptions, the following sets are in a natural correspondence given by bijections $\rho \rightarrow h_{\rho}^{\prime}$, and $\rho \rightarrow \chi=\operatorname{tr} \circ \rho$ :

- the set of all semisimple $S L_{n}$-representations of $G$;

- the set of all $R$-homomorphisms $\operatorname{Rep}_{n}^{R}(G)^{G L_{n}(R)} \rightarrow R$;

- the set of all $S L_{n}(R)$-characters of $G$.

By the proposition, we can identify the above sets and denote them by $X_{n}(G)$. By the second definition, $X_{n}(G)$ is the affine algebraic set composed of the closed points of the $S L_{n}$-character variety, $\mathfrak{X}_{n}(G)$, defined in the introduction. In other words,

$$
R\left[X_{n}(G)\right]=R\left[\mathfrak{X}_{n}(G)\right] / \sqrt{0}=\operatorname{Rep}_{n}^{R}(G)^{G L_{n}(R)} / \sqrt{0} .
$$

As shown in $[\mathrm{L}-\mathrm{M}] \overline{\mathrm{KM}}, \operatorname{Rep}_{n}^{R}(G)^{G L_{n}(R)}$ may contain nilpotent elements and, therefore, $\mathfrak{X}_{n}(G)$ contains more subtle information about $S L_{n}$-representations of $G$ than $X_{n}(G)$. The ring $\operatorname{Rep}_{n}^{R}(G)^{G L_{n}(R)}$ will be given a topological description in Section 3.

The $G L_{n}(R)$ action on $\operatorname{Rep} R(G)$ induces an action of $G L_{n}(R)$ on the ring of $n \times n$ matrices over $\operatorname{Rep}_{n}^{R}(G)$. If $M=\left(m_{i j}\right) \in M_{n}\left(\operatorname{Rep}_{n}^{R}(G)\right)$ and $A \in G L_{n}(R)$, then

$$
A * M=A\left(\begin{array}{cccc}
A * m_{11} & A * m_{12} & \ldots & A * m_{1 n} \\
\vdots & \vdots & \ldots & \vdots \\
A * m_{n 1} & A * m_{n 2} & \ldots & A * m_{n n}
\end{array}\right) A^{-1}
$$

There is an equivalent definition of the action of $G L_{n}(R)$ on $\operatorname{Re} p_{n}^{R}(G)$ and on $M_{n}\left(\operatorname{Re} p_{n}^{R}(G)\right)$. In order to introduce it we will first define $G L_{n}(R)$-actions on $P_{n}(I)$ and $M_{n}\left(P_{n}(I)\right)$. We can consider $P_{n}(I)$ as a ring of polynomial functions defined 
on the product of $I$ copies of $M_{n}(R), M_{n}(R)^{I} \rightarrow R$, by identifying $x_{j k}^{i_{0}} \in P_{n}(I)$ with a map assigning to $\left(M_{i}\right)_{i \in I} \in M_{n}(R)^{I}$ the $(j, k)$-entry of $M_{i_{0}}$. Therefore,

$$
P_{n}(I)=\operatorname{Poly}\left(M_{n}(R)^{I}, R\right)
$$

With this identification any entry in a matrix $M$ in $M_{n}\left(P_{n}(I)\right)$ is a polynomial function on $M_{n}(R)^{I}$. Therefore we can think of elements of $M_{n}\left(P_{n}(I)\right)$ as coordinatewise polynomial functions $M_{n}(R)^{I} \rightarrow M_{n}(R)$,

$$
M_{n}\left(P_{n}(I)\right)=\operatorname{Poly}\left(M_{n}(R)^{I}, M_{n}(R)\right) .
$$

If $X, Y$ are sets with a left $G$-action, then the set of all functions $F u n(X, Y)$ has a natural left $G$-action defined for any $f: X \rightarrow Y$ and $g \in G$ by $g * f(x)=$ $g f\left(g^{-1} x\right)$, for $x \in X . G L_{n}$ acts on $M_{n}(R)$ and on $M_{n}(R)^{I}$ by conjugation, and it acts trivially on $R$. These actions induce $G L_{n}(R)$-actions on $F u n\left(M_{n}(R)^{I}, R\right)$ and on $\operatorname{Fun}\left(M_{n}(R)^{I}, M_{n}(R)\right)$, which restrict to

$$
P_{n}(I)=\operatorname{Poly}\left(M_{n}(R)^{I}, R\right)
$$

and

$$
M_{n}\left(P_{n}(I)\right)=\operatorname{Poly}\left(M_{n}(R)^{I}, M_{n}(R)\right) .
$$

The following statement is a consequence of the above definitions.

Lemma 2.2. (1) The natural embedding of $P_{n}(I)$ into $M_{n}\left(P_{n}(I)\right)$ as scalar matrices is $G L_{n}(R)$-equivariant.

(2) $A_{i_{0}}=\left(x_{j k}^{i_{0}}\right) \in M_{n}\left(P_{n}(I)\right)$ is invariant under the action of $G L_{n}(R)$, for any $i_{0} \in I$.

Now, we are going to show that $\eta: P_{n}(I) \rightarrow \operatorname{Rep}_{n}^{R}(G)$ is $G L_{n}(R)$-equivariant, and hence the action of $G L_{n}(R)$ on $P_{n}(I)$ induces a $G L_{n}(R)$-action on $\operatorname{Rep}_{n}^{R}(G)$ which coincides with the $G L_{n}(R)$-action on $\operatorname{Rep}_{n}^{R}(G)$ defined previously.

Proposition 2.3. The following diagram commutes:

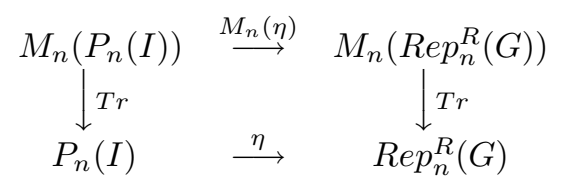

and all maps appearing in it intertwine with the $G L_{n}(R)$-action.

Proof. Since the commutativity of the above diagram is obvious, we will prove only that the trace functions and homomorphisms $\eta, M_{n}(\eta)$ are $G L_{n}(R)$-equivariant.

- The trace map $\operatorname{Tr}: M_{n}(R) \rightarrow R$ is $G L_{n}(R)$-equivariant. Therefore the induced map

$$
\operatorname{Tr}: M_{n}\left(P_{n}(I)\right)=\operatorname{Poly}\left(M_{n}(R)^{I}, M_{n}(R)\right) \rightarrow \operatorname{Poly}\left(M_{n}(R)^{I}, R\right)=P_{n}(I)
$$

is also $G L_{n}(R)$-equivariant.

- If $M=\left(m_{i j}\right) \in M_{n}\left(\operatorname{Rep}_{n}^{R}(G)\right)$ and $A \in G L_{n}(R)$, then $A * M$ is given by matrix (2.1), whose trace is $\operatorname{Tr}(A * M)=\sum_{i=1}^{n} A * m_{i i}=A * \operatorname{Tr}(M)$. Therefore

$$
\operatorname{Tr}: M_{n}\left(\operatorname{Rep}_{n}^{R}(G)\right) \rightarrow \operatorname{Rep}_{n}^{R}(G)
$$

is $G L_{n}(R)$-equivariant. 
- Recall that $P_{n}(I)$ is generated by elements $x_{j k}^{i}$. Therefore in order to prove that $\eta$ is $G L_{n}(R)$-equivariant it is enough to show that $\eta\left(A * x_{j k}^{i}\right)=A * \bar{x}_{j k}^{i}$, for any $i \in I, j, k \in\{1,2, \ldots, n\}$, where $\bar{x}_{j k}^{i}=\eta\left(x_{j k}^{i}\right) \in \operatorname{Rep}_{n}^{R} G$.

For any $i_{0} \in I, j_{G, n}\left(g_{i_{0}}\right)=\bar{A}_{i_{0}} \in S L_{n}\left(\operatorname{Rep}_{n}^{R}(G)\right)$. By the definition of the $G L_{n}(R)$-action on $\operatorname{Rep}_{n}^{R}(G), A^{-1} j_{G, n}\left(g_{i_{0}}\right) A$ is the matrix obtained from $j_{G, n}\left(g_{i_{0}}\right)$ by acting on all its entries by $A$. Therefore

$$
A * \bar{x}_{j k}^{i_{0}}=(j, k) \text {-entry of } A^{-1} \bar{A}_{i_{0}} A .
$$

Having described $A * \bar{x}_{j k}^{i_{0}}$, we need to give an explicit description of $A * x_{j k}^{i_{0}} \in$ $P_{n}(I)$. Recall that we identified $x_{j k}^{i_{0}}$ with the map $M_{n}(R)^{I} \rightarrow R$ assigning to $\left\{M_{i}\right\}_{i \in I}$ the $(j, k)$-entry of $M_{i_{0}}$. The definition of the $G L_{n}(R)$-action on maps between $G L_{n}(R)$-sets implies that

$$
\left(A * x_{j k}^{i_{0}}\right)\left(\left\{M_{i}\right\}_{i \in I}\right)=A *\left(x_{j k}^{i_{0}}\left(A^{-1} *\left\{M_{i}\right\}_{i \in I}\right)\right) .
$$

Since $G L_{n}(R)$ acts by simultaneous conjugation on $M_{n}(R)^{I}$ and it acts trivially on $R$, the right side of the above equation is equal to the $(j, k)$-entry of $A^{-1} M_{i_{0}} A$. But the entries of $M_{i_{0}}$ are given by the values of functions $x_{j k}^{i_{0}}$ evaluated on $\left\{M_{i}\right\}_{i \in I}$. Therefore

$$
A * x_{j k}^{i_{0}}=(j, k) \text {-entry of } A^{-1}\left(x_{j k}^{i_{0}}\right) A .
$$

Finally, (2.3) and (2.4) imply that

$$
\begin{gathered}
\eta\left(A * x_{j k}^{i_{0}}\right)=\eta\left(\text { the }(j, k) \text {-entry of } A^{-1} A_{i_{0}} A\right) \\
=\text { the }(j, k) \text {-entry of } A^{-1} \bar{A}_{i_{0}} A=A * \bar{x}_{j k}^{i_{0}} .
\end{gathered}
$$

- We prove that $M_{n}(\eta)$ is equivariant. Let $M=\left(m_{j k}\right) \in M_{n}\left(P_{n}(I)\right)$. Notice that the definition of $G L_{n}(R)$-action on $M_{n}\left(P_{n}(I)\right)$ implies that $A * M=$ $A\left(A * m_{j k}\right) A^{-1}$. Therefore

$$
M_{n}(\eta)(A * M)=M_{n}(\eta)\left(A\left(A * m_{j k}\right) A^{-1}\right)=A\left(\eta\left(A * m_{j k}\right)\right) A^{-1} .
$$

Since $\eta$ is $G L_{n}(R)$-equivariant, the matrix on the right side of the above equation is $A\left(A * \eta\left(m_{j k}\right)\right) A^{-1}=A *\left(\eta\left(m_{j k}\right)\right)$. Therefore $M_{n}(\eta)$ is also $G L_{n}(R)$ equivariant.

The above proposition implies that there exists a function

$$
\operatorname{Tr}: M_{n}\left(\operatorname{Rep}_{n}^{R}(G)\right)^{G L_{n}(R)} \rightarrow \operatorname{Rep}_{n}^{R}(G)^{G L_{n}(R)} .
$$

This function will be given a simple topological interpretation in the next section.

Proposition 2.4. The image of the universal representation

$$
j_{G, n}: G \rightarrow M_{n}\left(\operatorname{Rep}_{n}^{R}(G)\right)
$$

is invariant under the action of $G L_{n}(R)$.

Proof. Since the elements $g_{i}$ generate $G$, it is sufficient to show that $j_{G, n}\left(g_{i}\right) \in$ $M_{n}\left(\operatorname{Rep}_{n}^{R}(G)\right)^{G L_{n}(R)}$. By Lemma 2.2(2), $A_{i} \in M_{n}\left(P_{n}(I)\right)^{G L_{n}(R)}$. The map $M_{n}(\eta)$ is equivariant. Therefore it takes the invariant $A_{i}$ to the invariant $M_{n}(\eta)\left(A_{i}\right)=$ $j_{G, n}\left(g_{i}\right)$. 


\section{Skein ALGEBRAS}

In this section we assign to each path-connected topological space $X$ a commutative $R$-algebra $\mathbb{A}_{n}(X)$ and to each pointed path-connected topological space $\left(X, x_{0}\right)$ an $R$-algebra $\mathbb{A}_{n}\left(X, x_{0}\right)$. These algebras encode the most important information about the $S L_{n}$-representations of $\pi_{1}\left(X, x_{0}\right)$. We will show that if $R$ is a field of characteristic 0 (but not necessarily algebraically closed), then $\mathbb{A}_{n}(X)$ is isomorphic to the universal character ring $\operatorname{Rep}_{n}^{R}(G)^{G L_{n}(R)}$, where $G=\pi_{1}\left(X, x_{0}\right)$, and $\mathbb{A}_{n}\left(X, x_{0}\right)$ is isomorphic to $M_{n}\left(\operatorname{Rep}_{n}^{R}(G)\right)^{G L_{n}(R)}$.

We start with a definition of a graph which is the most suitable for our purposes. A graph $D=(\mathcal{V}, \mathcal{E}, \mathcal{L})$ consists of a vertex-set $\mathcal{V}$, a set of oriented edges $\mathcal{E}$, and a set of oriented loops $\mathcal{L}$. Each edge $E \in \mathcal{E}$ has a beginning $b(E) \in \mathcal{V}$ and an end $e(E) \in \mathcal{V}$. Loops have neither beginnings nor ends. If $b(E)=v$ or $e(E)=v$, then $E$ is incident with $v$. For any $v \in \mathcal{V}$, all edges incident to $v$ are ordered by consecutive integers $1,2, \ldots$. Therefore the beginning and the end of each edge is assigned a number.

The sets $\mathcal{V}, \mathcal{E}, \mathcal{L}$ are finite. We topologize each graph as a $\mathrm{CW}$-complex. The topology of a graph coincides with the topology of its edges $E \simeq[0,1], E \in \mathcal{E}$, and its loops $L \simeq S^{1}, L \in \mathcal{L}$. There is a natural notion of isomorphism of graphs.

Let $\mathcal{G}$ be a set of representatives of all isomorphism classes of graphs defined above. We say that a vertex $v$ is an $n$-valent source of a graph $D$ if $n$ distinct edges of $D$ begin at $v$ and no edge ends at $v$. Similarly, we say that $v$ is an $n$-valent $\operatorname{sink}$ of $D$ if $n$ distinct edges end at $v$ and no edge begins at $v$. Let $\mathcal{G}_{n}$ denote the set of all graphs in $\mathcal{G}$, all of whose vertices are either $n$-valent sources or $n$-valent sinks. We assume that the empty graph $\emptyset$ is also an element of $\mathcal{G}_{n}$. We denote the single loop in $\mathcal{G}_{n}$, i.e. the connected graph without any vertices, by $S^{1}$. Let $\mathcal{G}_{n}^{\prime}$ denote the set of all graphs $D \in \mathcal{G}$ such that $D$ has one 1-valent source and one 1-valent sink, and all other vertices of $D$ are $n$-valent sources or $n$-valent sinks. We denote the single edge in $\mathcal{G}_{n}^{\prime}$, i.e. the connected graph without any $n$-valent vertices, by $[0,1]$.

Let $X$ be a path-connected topological space. We will call any continuous map $f: D \rightarrow X$, where $D \in \mathcal{G}_{n}$, a graph in $X$. We identify two maps $f_{1}, f_{2}: D \rightarrow X$ if they are homotopic. Let us denote the set of all graphs in $X$ by $\mathcal{G}_{n}(X)$. Similarly, we define $\mathcal{G}_{n}\left(X, x_{0}\right)$ to be the set of all maps $f: D \rightarrow X \times[0,1]$, where $D \in \mathcal{G}_{n}^{\prime}$ and $f$ maps the 1 -valent sink of $D$ to $\left(x_{0}, 1\right)$ and the 1 -valent source of $D$ to $\left(x_{0}, 0\right)$. We identify maps which are homotopic relative to $\left(x_{0}, 0\right)$ and $\left(x_{0}, 1\right)$. We will call elements of $\mathcal{G}_{n}\left(X, x_{0}\right)$ relative graphs in $X \times[0,1]$.

We introduce a few classes of graphs in $\mathcal{G}_{n}(X)$ and $\mathcal{G}_{n}\left(X, x_{0}\right)$ which will often be used later on in the paper. Let $L_{\gamma}: S^{1} \rightarrow X$ be a graph in $X$ which represents the conjugacy class of $\gamma \in \pi_{1}\left(X, x_{0}\right)$. We denote by $E_{\gamma}$ a relative graph $E_{\gamma}:[0,1] \rightarrow$ $X \times[0,1], E_{\gamma}(0)=\left(x_{0}, 0\right), E_{\gamma}(1)=\left(x_{0}, 1\right)$, whose projection into $X$,

$$
[0,1] \stackrel{E_{\gamma}}{\rightarrow} X \times[0,1] \rightarrow X,
$$

represents $\gamma \in \pi_{1}\left(X, x_{0}\right)$. Let $E L_{\gamma}:[0,1] \cup S^{1} \rightarrow X \times[0,1]$ be a relative graph such that $E L_{\gamma}(t)=\left(x_{0}, t\right)$ for $t \in[0,1]$, and $E L_{\gamma \mid S^{1}}: S^{1} \rightarrow X \times[0,1] \rightarrow X$ represents the conjugacy class of $\gamma \in \pi_{1}\left(X, x_{0}\right)$.

For any two graphs $f_{1}: D_{1} \rightarrow X$ and $f_{2}: D_{2} \rightarrow X, f_{1}, f_{2} \in \mathcal{G}_{n}(X)$, we define a product of them to be $f_{1} \cup f_{2}: D_{1} \cup D_{2} \rightarrow X, f_{1} \cup f_{2} \in \mathcal{G}_{n}(X)$, where $D_{1} \cup D_{2}$ denotes the disjoint union of $D_{1}$ and $D_{2}$. Therefore the free $R$-module $R \mathcal{G}_{n}(X)$ on 
$\mathcal{G}_{n}(X)$ can be considered as a commutative $R$-algebra. The empty graph $\emptyset: \emptyset \rightarrow X$ is an identity in $R \mathcal{G}_{n}(X)$.

In the next definition we will represent fragments of diagrams by coupons, as depicted below:

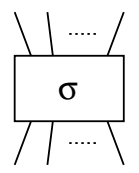

This coupon means a braid corresponding to a permutation $\sigma \in S_{n}$ 1]

Example 3.1. If $\sigma=(1,2,3) \in S_{3}$, then

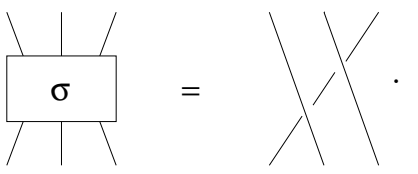

Suppose that $f: D \rightarrow X$ is a graph in $X$ and $f$ maps a source $w$ and a sink $v$ of $D$ to the same point $x_{1} \in X$. Let $D_{\sigma}$ be a graph obtained from $D$ by replacing

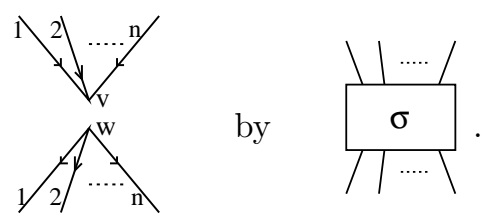

In the diagram we display $v$ and $w$ as separate points to accentuate the fact that they are distinct in the domain of the mapping $f$. There is an obvious way to modify $f: D \rightarrow X$ to a map $f_{\sigma}: D_{\sigma} \rightarrow X$. We call $\left(f,\left\{f_{\sigma}\right\}_{\sigma \in S_{n}}\right)$ a family of skein related graphs at $x_{1}$.

Definition 3.2. Let $X$ be a path-connected topological space, and let $I$ be the ideal in $R \mathcal{G}_{n}(X)$ generated by two kinds of expressions:

(1) $f-\sum_{\sigma \in S_{n}} \epsilon(\sigma) f_{\sigma}$, where $\epsilon(\sigma)$ denotes the sign of $\sigma$ and $\left(f,\left\{f_{\sigma}\right\}_{\sigma \in S_{n}}\right)$ is a family of skein related graphs at some point $x_{1} \in X$.

(2) $L_{e}-n$, where $e$ is the identity element in $\pi_{1}\left(X, x_{0}\right)$ (i.e. $L_{e}$ is a homotopically trivial loop).

Then the $R$-algebra $\mathbb{A}_{n}(X)=R \mathcal{G}_{n}(X) / I$ is called the $n$-th skein algebra of $X$.

Similarly we define $\mathbb{A}_{n}\left(X, x_{0}\right)$. Let $f_{1}: D_{1} \rightarrow X \times[0,1], f_{2}: D_{2} \rightarrow X \times[0,1]$ be elements of $\mathcal{G}_{n}\left(X, x_{0}\right)$. We define the product of them to be a map $f_{1} \cdot f_{2}$ : $D_{1} \cup D_{2} \rightarrow X \times[0,1]$, such that

$$
\left(f_{1} \cdot f_{2}\right)(d)= \begin{cases}\left(x, \frac{1}{2} t\right) & \text { if } d \in D_{1} \text { and } f_{1}(d)=(x, t), \\ \left(x, \frac{1}{2} t+\frac{1}{2}\right) & \text { if } d \in D_{2} \text { and } f_{2}(d)=(x, t) .\end{cases}
$$

This product extends to an associative (but generally non-commutative) product in $R \mathcal{G}_{n}\left(X, x_{0}\right)$. The identity in $R \mathcal{G}_{n}\left(X, x_{0}\right)$ is a map $f: E \rightarrow X \times[0,1]$, where $E$ is a single edge and $f$ maps $E$ onto $\left\{x_{0}\right\} \times[0,1]$.

${ }^{1}$ Since we consider graphs up to homotopy equivalence, it does not matter which braid corresponding to $\sigma$ we take. 
If $f: D \rightarrow X \times[0,1]$ is an element of $\mathcal{G}_{n}\left(X, x_{0}\right)$ such that an $n$-valent source $w$ and an $n$-valent sink $v$ of $D$ are mapped to a point $x_{1} \in X \times[0,1]$, then one can define $D_{\sigma}$ and $f_{\sigma}: D_{\sigma} \rightarrow X \times[0,1], f_{\sigma} \in \mathcal{G}_{n}\left(X, x_{0}\right)$, in exactly the same way as it was done for graphs in $\mathcal{G}_{n}(X)$ in the paragraph preceding Definition 3.2 We say, as before, that $\left(f,\left\{f_{\sigma}\right\}_{\sigma \in S_{n}}\right)$ are graphs skein related at $x_{1}$.

Definition 3.3. Let $X$ be a path-connected topological space with a specified point $x_{0} \in X$, and let $I^{\prime}$ be the ideal in $R \mathcal{G}_{n}\left(X, x_{0}\right)$ generated by expressions

(1) $f-\sum_{\sigma \in S_{n}} \epsilon(\sigma) f_{\sigma}$, where $\left(f,\left\{f_{\sigma}\right\}_{\sigma \in S_{n}}\right)$ is a family of skein related graphs at some point $x_{1} \in X \times[0,1]$.

(2) $E L_{e}-n$, where $e$ is the identity in $\pi_{1}\left(X, x_{0}\right)$.

Then the $R$-algebra $\mathbb{A}_{n}\left(X, x_{0}\right)=R \mathcal{G}_{n}\left(X, x_{0}\right) / I^{\prime}$ is called the $n$-th relative skein algebra of $\left(X, x_{0}\right)$.

Note that different choices of $x_{0} \in X$ give isomorphic algebras $\mathbb{A}_{n}\left(X, x_{0}\right)$.

Let $f \in \mathcal{G}_{n}(X), f: D \rightarrow X$. Let $D^{\prime}=D \cup E$, where $E$ is an edge disjoint from $D$. Then $D^{\prime}$ has one 1 -valent sink $e_{0}$ and one 1 -valent source $e_{1},\left\{e_{0}, e_{1}\right\}=\partial E$, and $D^{\prime} \in \mathcal{G}_{n}^{\prime}$. We extend $f$ to $f^{\prime}: D^{\prime} \rightarrow X \times[0,1]$ in such a way that $f^{\prime}(d)=$ $\left(f(d), \frac{1}{2}\right)$ for $d \in D$, and $f^{\prime}(t)=\left(x_{0}, t\right)$ for $t \in[0,1] \simeq E$, where $[0,1] \simeq E$ is an orientation-preserving parameterization of $E$. This operation defines an embedding $\imath: \mathcal{G}_{n}(X) \rightarrow \mathcal{G}_{n}\left(X, x_{0}\right), \imath(f)=f^{\prime}$. Notice that $\imath$ induces a homomorphism $\imath_{*}$ : $\mathbb{A}_{n}(X) \rightarrow \mathbb{A}_{n}\left(X, x_{0}\right)$. Therefore we can consider $\mathbb{A}_{n}\left(X, x_{0}\right)$ as an $\mathbb{A}_{n}(X)$-algebra.

Let $f: D \rightarrow X \times[0,1]$ be a map, $f \in \mathcal{G}_{n}\left(X, x_{0}\right)$. Let $\bar{D} \in \mathcal{G}_{n}$ be a graph obtained by identification of the 1-valent sink with the 1-valent source in $D$. Let us compose $f: D \rightarrow X \times[0,1]$ with a projection $X \times[0,1] \rightarrow X$. This composition gives a map $\bar{f}: \bar{D} \rightarrow X, \bar{f} \in \mathcal{G}_{n}(X)$. Therefore, we have a function ${ }^{-}: \mathcal{G}_{n}\left(X, x_{0}\right) \rightarrow \mathcal{G}_{n}(X)$. This function can be extended to an $R$-linear homomorphism $\mathbb{T}: \mathbb{A}_{n}\left(X, x_{0}\right) \rightarrow \mathbb{A}_{n}(X)$. Notice that for any graph $D \in \mathcal{G}_{n}(X), \mathbb{T}\left(\imath_{*}(D)\right)$ is equal to a union of $f: D \rightarrow X$ with a contractible loop in $X$. Hence by Definition $3.2(2) \mathbb{T}\left(\imath_{*}(D)\right)=n \cdot D$. Since graphs in $X$ span $\mathbb{A}_{n}(X)$, the composition of $\imath_{*}: \mathbb{A}_{n}(X) \rightarrow \mathbb{A}_{n}\left(X, x_{0}\right)$ with $\mathbb{T}$ : $\mathbb{A}_{n}\left(X, x_{0}\right) \rightarrow \mathbb{A}_{n}(X)$ is equal to $n$ times the identity on $\mathbb{A}_{n}(X)$. This implies the following fact.

Fact 3.4. If $\frac{1}{n} \in R$, then $\imath_{*}: \mathbb{A}_{n}(X) \rightarrow \mathbb{A}_{n}\left(X, x_{0}\right)$ is a monomorphism of rings.

The next proposition summarizes basic properties of $\mathbb{A}_{n}(X)$ and $\mathbb{A}_{n}\left(X, x_{0}\right)$.

Proposition 3.5. (1) The assignment $X \rightarrow \mathbb{A}_{n}(X)$ (respectively, $\left(X, x_{0}\right) \rightarrow$ $\left.\mathbb{A}_{n}\left(X, x_{0}\right)\right)$ defines a functor from the category of path-connected topological spaces (respectively, category of path-connected pointed spaces) to the category of commutative $R$-algebras (respectively, the category of $R$-algebras).

(2) If $f: X \rightarrow Y, f\left(x_{0}\right)=y_{0}$, induces a surjection $f_{*}: \pi_{1}\left(X, x_{0}\right) \rightarrow \pi_{1}\left(Y, y_{0}\right)$, then the corresponding homomorphisms $\mathbb{A}_{n}(f): \mathbb{A}_{n}\left(X, x_{0}\right) \rightarrow \mathbb{A}_{n}\left(Y, y_{0}\right)$ and $\mathbb{A}_{n}(f): \mathbb{A}_{n}(X) \rightarrow \mathbb{A}_{n}(Y)$ are epimorphisms of $R$-algebras.

(3) The algebra $\mathbb{A}_{n}(X)$ is generated by loops in $X$, i.e. by graphs $L_{\gamma}$, for $\gamma \in$ $\pi_{1}\left(X, x_{0}\right)$.

(4) The algebra $\mathbb{A}_{n}\left(X, x_{0}\right)$ is generated by graphs $E_{g_{i}^{ \pm 1}}$ and $E L_{\gamma}$, where $\left\{g_{i}\right\}_{i \in I}$ is a set of generators of $\pi_{1}\left(X, x_{0}\right)$ and $\gamma \in \pi_{1}\left(X, x_{0}\right)$.

Proof. Since statements (1) and (2) of Proposition 3.5 are obvious, we give a proof of (3) and (4) only. 
From the definition of a graph $D \in \mathcal{G}_{n}$ or $D \in \mathcal{G}_{n}^{\prime}$ we see that it has an equal number of $n$-valent sinks and sources. Relation (1) of Definition 3.2 and of Definition 3.3 implies that each pair of vertices of $f: D \rightarrow X, f \in \mathcal{G}_{n}(X)$ (respectively, of $\left.f: D \rightarrow X \times[0,1], f \in \mathcal{G}_{n}\left(X, x_{0}\right)\right)$ composed of a sink and a source can be resolved and $f$ can be replaced by a linear combination of graphs with a smaller number of sinks and sources. Therefore, after a finite number of steps each graph in $\mathcal{G}_{n}(X)$ (respectively, $\mathcal{G}_{n}\left(X, x_{0}\right)$ ) can be expressed as a linear combination of graphs without $n$-valent vertices.

(3) If $f: D \rightarrow X, f \in \mathcal{G}_{n}(X)$, and $D$ has no vertices, then $D$ is a union of loops, $D=S^{1} \cup S^{1} \cup \ldots \cup S^{1}$, and therefore $f=L_{\gamma_{1}} \cdot L_{\gamma_{2}} \cdot \ldots \cdot L_{\gamma_{k}} \in \mathbb{A}_{n}(X)$, for some $\gamma_{1}, \gamma_{2}, \ldots, \gamma_{k} \in \pi_{1}\left(X, x_{0}\right)$.

(4) If $f: D \rightarrow X \times[0,1], f \in \mathcal{G}_{n}\left(X, x_{0}\right)$, and $D$ has no $n$-valent vertices, then $D=[0,1] \cup S^{1} \cup S^{1} \cup \ldots \cup S^{1}$. Suppose that $[0,1] \stackrel{f}{\rightarrow} X \times[0,1] \rightarrow X$ represents $\gamma_{0} \in \pi_{1}\left(X, x_{0}\right)$, and $f$ restricted to the $j$-th circle represents the conjugacy class of a $\gamma_{j} \in \pi_{1}\left(X, x_{0}\right), j=1,2, \ldots, k$. Then $f=E_{\gamma_{0}} \cdot E L_{\gamma_{1}} \cdot E L_{\gamma_{2}} \cdot \ldots \cdot E L_{\gamma_{k}} \in \mathbb{A}_{n}\left(X, x_{0}\right)$. Therefore $\mathbb{A}_{n}\left(X, x_{0}\right)$ is generated by the elements $E L_{\gamma}$ and $E_{\gamma^{\prime}}, \gamma, \gamma^{\prime} \in \pi_{1}\left(X, x_{0}\right)$. But each $E_{\gamma^{\prime}}$ is a product of elements $E_{g_{i}^{ \pm 1}}$, where $\left\{g_{i}\right\}$ is a set of generators of $\pi_{1}\left(X, x_{0}\right)$.

We will see later that $\mathbb{A}_{n}(X)$ and $\mathbb{A}_{n}\left(X, x_{0}\right)$ depend only on $\pi_{1}\left(X, x_{0}\right)$. Moreover, if $\pi_{1}\left(X, x_{0}\right)$ is a finitely generated group, then the algebras $\mathbb{A}_{n}(X)$ and $\mathbb{A}_{n}\left(X, x_{0}\right)$ are also finitely generated.

Now we are ready to formulate the most important results of this paper.

Theorem 3.6. Let $X$ be any (path-connected) topological space, and let $G=$ $\pi_{1}\left(X, x_{0}\right), x_{0} \in X$. There are $R$-algebra homomorphisms

$$
\Theta: \mathbb{A}_{n}\left(X, x_{0}\right) \rightarrow M_{n}\left(\operatorname{Rep}_{n}^{R}(G)\right)^{G L_{n}(R)}, \quad \theta: \mathbb{A}_{n}(X) \rightarrow \operatorname{Rep}_{n}^{R}(G)^{G L_{n}(R)},
$$

uniquely determined by the following conditions:

(1) $\Theta\left(E_{\gamma}\right)=j_{G, n}(\gamma)$ and $\Theta\left(E L_{\gamma^{\prime}}\right)=\operatorname{Tr}\left(j_{G, n}\left(\gamma^{\prime}\right)\right)$, for any $\gamma, \gamma^{\prime} \in \pi_{1}\left(X, x_{0}\right)$.

(2) $\theta\left(L_{\gamma}\right)=\operatorname{Tr}\left(j_{G, n}(\gamma)\right)$, for any $\gamma \in \pi_{1}\left(X, x_{0}\right)$.

Moreover, the following diagram commutes:

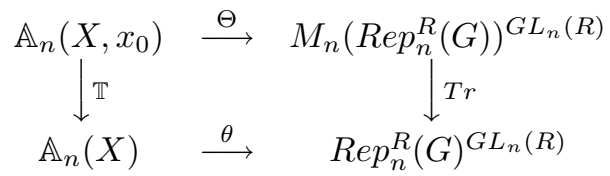

Theorem 3.7. If $R$ is a field of characteristic 0 , then

$$
\Theta: \mathbb{A}_{n}\left(X, x_{0}\right) \rightarrow M_{n}\left(\operatorname{Rep}_{n}^{R}(G)\right)^{G L_{n}(R)}, \quad \theta: \mathbb{A}_{n}(X) \rightarrow \operatorname{Rep}_{n}^{R}(G)^{G L_{n}(R)}
$$

are isomorphisms of $R$-algebras.

Let $R$ be a field of characteristic 0 . It can be shown that if $X$ is a 3-manifold, then $\mathbb{A}_{2}(X)$ is isomorphic to the Kauffman bracket skein module of $X, \mathcal{S}_{2, \infty}(X, R, \pm 1)$. Moreover, if $X$ is a surface, then $\mathbb{A}_{2}\left(X, x_{0}\right)$ is isomorphic to the relative Kauffman bracket skein module of $X, \mathcal{S}_{2, \infty}^{r e l}(X, R, \pm 1)$. See [PS-2], [H-P], for appropriate definitions and the notational conventions. The main results of [B-1], [B-2], [PS-1] 
and PS-2 relate the Kauffman bracket skein modules of 3-manifolds with the $S L_{2}$ representation theory of their fundamental groups. Theorem 3.7 generalizes these results to groups $S L_{n}$, for any $n$.

Moreover, it can be shown that in the case when $X$ is any path-connected topological space, $\mathbb{A}_{2}\left(X, x_{0}\right)$ and $\mathbb{A}_{2}(X)$ can be given the following simple algebraic description: Let $G=\pi_{1}(X)$ and let $I$ be the ideal in the group ring $R G$ generated by elements $h\left(g+g^{-1}\right)-\left(g+g^{-1}\right) h$, where $g, h \in G$. There is an involution $\tau$ on $H(G)=R G / I$ sending $g$ to $g^{-1}$. One can show that $\mathbb{A}_{2}\left(X, x_{0}\right)$ is isomorphic to $H(G)$ and $\mathbb{A}_{2}(X)$ is isomorphic to $H^{+}(G)$, where $H^{+}(G)$ is the subring of $H(G)$ invariant under $\tau$. The algebras $H(G), H^{+}(G)$ are introduced and thoroughly investigated in $[\mathrm{B}-\mathrm{H}]$. One of the main results of $[\mathrm{B}-\mathrm{H}]$ is that $H(G)=M_{n}\left(\operatorname{Rep}_{n}^{R}(G)\right)^{G L_{n}(R)}$ and $H^{+}(G)=R e p_{n}^{R}(G)^{G L_{n}(R)}$, for $n=2$. (Compare also [Sa-1], Sa-2].) Theorem 3.7 can be considered as a generalization of this result to all values of $n$.

\section{Proof of Theorem 3.6}

Before we prove Theorem 3.6 we give new definitions of $\mathbb{A}_{n}(X)$ and $\mathbb{A}_{n}\left(X, x_{0}\right)$ which only use $G=\pi_{1}\left(X, x_{0}\right)$.

Let $X$ be a path-connected topological space and $x_{0} \in X$. For any graph in $\mathcal{G}_{n}(X)$, i.e. a map $f: D \rightarrow X$ for some $D \in \mathcal{G}_{n}$, there is a map $f^{\prime}: D \rightarrow X$ homotopic to $f$, which maps all vertices of $D$ to $x_{0}$. Therefore the homotopy class of $f$ can be described by the graph $D$ with each edge $E$ labeled by an element of $\pi_{1}\left(X, x_{0}\right)$ corresponding to the map $f_{\mid E}^{\prime}: E \rightarrow X$ and each loop $L$ labeled by the conjugacy class in $\pi_{1}\left(X, x_{0}\right)$ corresponding to the map $f_{\mid L}^{\prime}: L \simeq S^{1} \stackrel{f}{\rightarrow} X$. This description does not need to be unique.

We denote the set of graphs in $\mathcal{G}_{n}$ all of whose edges are labeled by elements of $G$ and all loops are labeled by conjugacy classes in $G$ by $\mathcal{G}_{n}(G)$. There is a natural multiplication operation on $\mathcal{G}_{n}(G)$. The product of $D_{1}, D_{2} \in \mathcal{G}_{n}(G)$ is the disjoint union of $D_{1}$ and $D_{2}$. Therefore $R \mathcal{G}_{n}(G)$ is a commutative $R$-algebra with $\emptyset$ as the identity.

Let $D$ be a graph in $\mathcal{G}_{n}(G)$. We have noticed already that $D$ corresponds to a map $f: D \rightarrow X$ which maps all vertices of $D$ to $x_{0}$ and restricted to edges and loops of $D$ agrees with their labeling. Such $f$ is unique up to a homotopy which fixes the vertices of $D$. Let $w$ be a source and $v$ be a sink in $D$. Since $f$ maps $v$ and $w$ to the same point in $X$, there exists a map $f_{\sigma}: D_{\sigma} \rightarrow X$ defined for any $\sigma \in S_{n}$ as in the paragraph preceding Definition 3.2 Notice that $f_{\sigma}$ maps all vertices of $D_{\sigma}$ to $x_{0} \in X$. Therefore, we can label all edges of $D_{\sigma}$ by appropriate elements of $G$ and all loops of $D_{\sigma}$ by appropriate conjugacy classes in $G$, and hence consider $D_{\sigma}$ as an element of $\mathcal{G}_{n}(G)$. Hence, we have showed that one can replace any source $w$ and any sink $v$ in an arbitrary graph $D \in \mathcal{G}_{n}(G)$

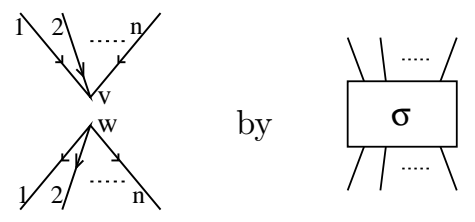

and obtain a well-defined graph $D_{\sigma} \in \mathcal{G}_{n}(G)$. 
As an example consider the graph $D$ presented below:

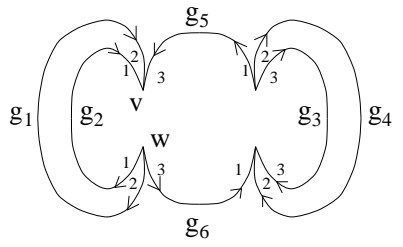

Replacing the vertices $v, w$ by a coupon decorated by $\sigma=(123) \in S_{3}$ gives a diagram $D_{\sigma}$ :

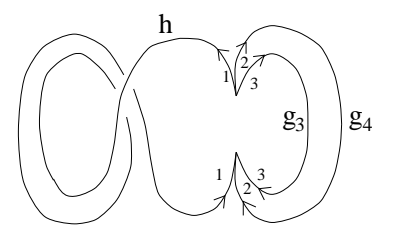

where $h=g_{6} g_{1} g_{2} g_{5}$.

Now we are ready to define $\mathbb{A}_{n}(X)$ in terms of graphs in $\mathcal{G}_{n}(G)$. Namely, this algebra is isomorphic to $R \mathcal{G}_{n}\left(\pi_{1}\left(X, x_{0}\right)\right) / I$, where $I \triangleleft R \mathcal{G}_{n}\left(\pi_{1}\left(X, x_{0}\right)\right)$ is an ideal generated by relations analogous to relations (1) and (2) of Definition 3.2 and by relations following from the fact that the assignment $\mathcal{G}_{n}(G) \rightarrow \mathcal{G}_{n}(X)$ described above is onto but not 1-1. The problem comes from the fact that one can take a graph $D \in \mathcal{G}_{n}$ whose edges and loops are labeled in two different ways such that the corresponding maps $f, f^{\prime}: D \rightarrow X$ sending the vertices of $D$ to $x_{0}$ are homotopic but not by a homotopy relative to the vertices of $D$. In order to resolve this problem we need to allow an operation which moves vertices of $D$ around paths in $X$ beginning and ending at $x_{0}$. Notice however that it suffices to move one vertex at a time. The following fact summarizes our observations.

Fact 4.1. Let $X$ be a path-connected topological space with a specified point $x_{0} \in X$, and let $G=\pi_{1}\left(X, x_{0}\right)$. Let $I$ be the ideal in $R \mathcal{G}_{n}(G)$ generated by expressions of the following form:
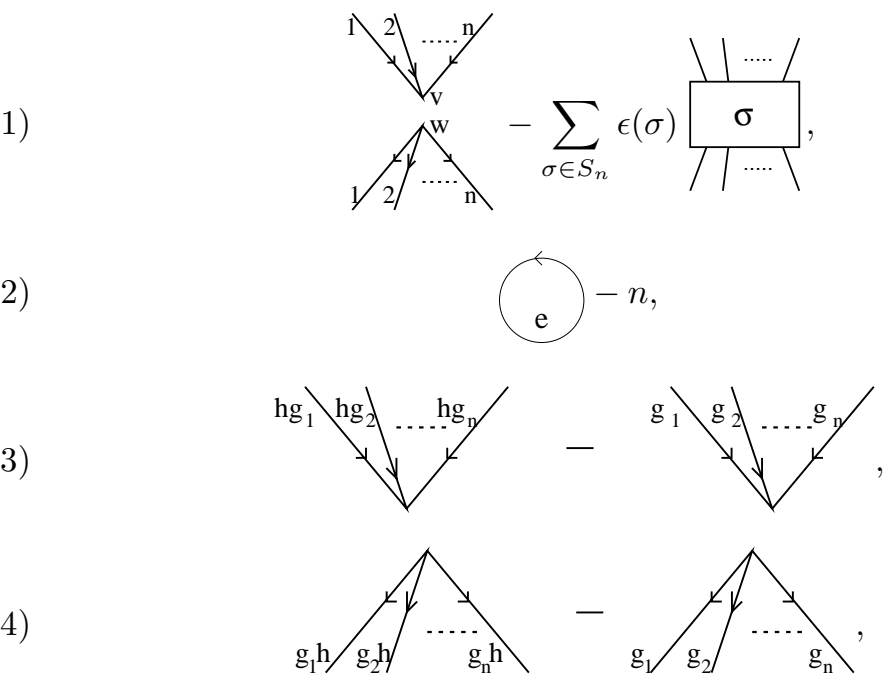

for any $g_{1}, g_{2}, \ldots, g_{n}, h \in G$. 
Then there is an isomorphism between the $R$-algebras $\mathbb{A}_{n}(X)$ and $R \mathcal{G}_{n}(G) / I$ assigning to each graph $f: D \rightarrow X, f \in \mathcal{G}_{n}(X)$, with all vertices at $x_{0}$ the graph $D$ with every edge $E$ of $D$ decorated by the element of $\pi_{1}\left(X, x_{0}\right)$ corresponding to $f_{\mid E}: E \rightarrow X$, and every loop $L$ of $D$ decorated by the conjugacy class in $\pi_{1}\left(X, x_{0}\right)$ represented by $f_{\mid L}: L \rightarrow X$.

Now we will state a similar fact for $\mathbb{A}_{n}\left(X, x_{0}\right)$. Let $\mathcal{G}_{n}^{\prime}(G)$ be a set of graphs in $\mathcal{G}_{n}^{\prime}$ all of whose edges are labeled by elements of $G$ and all of whose loops are labeled by conjugacy classes in $G$.

There is a multiplication operation defined on $\mathcal{G}_{n}^{\prime}(G)$ in the following way. Let $D_{1}, D_{2} \in \mathcal{G}_{n}^{\prime}(G)$, let $v_{i}$ be the 1-valent source of $D_{i}, i \in\{1,2\}$, and let $w_{i}$ be the 1 -valent sink of $D_{i}$. Let $g_{i}$ be the label of the edge of $D_{i}$ joining $v_{i}$ with $w_{i}$. The graph $D_{1} \cdot D_{2}$ is obtained from the disjoint union of $D_{1}$ and $D_{2}$ by identifying $v_{1}$ with $w_{2}$. The edge of $D_{1} \cdot D_{2}$ joining $v_{2}$ with $w_{1}$ is labeled by $g_{1} \cdot g_{2}$. All other edges and loops of $D_{1} \cdot D_{2}$ inherit labels from $D_{1}$ and $D_{2}$. A single edge labeled by $e \in G$ is the identity in $\mathcal{G}_{n}^{\prime}(G)$.

This multiplication extends to an associative (but not commutative) multiplication in $R \mathcal{G}_{n}^{\prime}(G)$.

Fact 4.2. Let $X$ be a path-connected topological space with a specified point $x_{0} \in X$, and let $G=\pi_{1}\left(X, x_{0}\right)$. Let $I^{\prime}$ be the ideal in $R \mathcal{G}_{n}^{\prime}(G)$ generated by the expressions (4.1), (4.3), (4.4) and

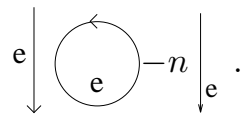

Then $\mathbb{A}_{n}\left(X, x_{0}\right) \simeq R \mathcal{G}_{n}^{\prime}(G) / I^{\prime}$.

Facts 4.1 and 4.2 show that the algebras $\mathbb{A}_{n}\left(X, x_{0}\right)$ and $\mathbb{A}_{n}(X)$ depend only on $\pi_{1}\left(X, x_{0}\right)$. In fact 4.1 and 4.2 give us models for $\mathbb{A}_{n}\left(X, x_{0}\right)$ and $\mathbb{A}_{n}(X)$ built from $\mathcal{G}_{n}(G)$ and $\mathcal{G}_{n}^{\prime}(G)$. In the rest of this section we will use these models.

Let us fix a commutative ring $R$ and a positive integer $n$ and a topological space $X$ with $x_{0} \in X, \pi_{1}\left(X, x_{0}\right)=G$. Let $\mathcal{R}=\operatorname{Rep}_{n}^{R}(G)$ and let $V=\mathcal{R}^{n}$ be a free $n$-dimensional module over $\mathcal{R}$ with the standard basis, $\left\{e_{1}, e_{2}, \ldots, e_{n}\right\}, e_{i}=$ $(0,0, \ldots, 1, \ldots, 0)$. The dual space $V^{*}$ has the dual basis $e^{1}, e^{2}, \ldots, e^{n}, e^{i}\left(e_{j}\right)=\delta_{i, j}$. We will always use the standard bases and therefore identify $V^{*} \otimes V \simeq \operatorname{End}_{\mathcal{R}}(V) \simeq$ $M_{n}(\mathcal{R})$.

Let $D$ be an element of $\mathcal{G}_{n}(G)$ or $\mathcal{G}_{n}^{\prime}(G)$. We can decompose $D$ into arcs, sources and sinks:

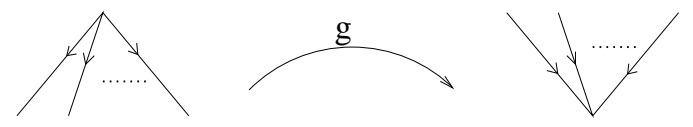

\section{Example 4.3.}

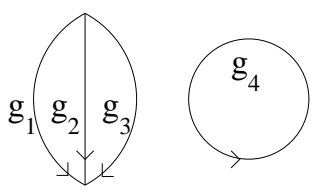


can be decomposed to

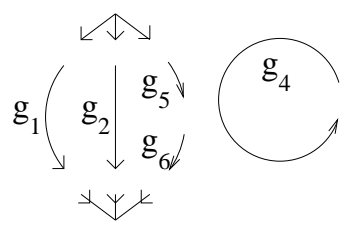

where $g_{1}, g_{2}, g_{3}, g_{4}, g_{5}, g_{6} \in G, g_{3}=g_{6} g_{5}$.

Notice that the decomposition of a graph is not unique, since we can cut each edge or loop into many pieces.

Let us assign to each $n$-valent source the tensor

$$
\sum_{\sigma \in S_{n}} \epsilon(\sigma) e_{\sigma(1)} \otimes e_{\sigma(2)} \otimes \ldots \otimes e_{\sigma(n)} \in V^{\otimes n}
$$

and to each $n$-valent sink the tensor

$$
\sum_{\sigma \in S_{n}} \epsilon(\sigma) e^{\sigma(1)} \otimes e^{\sigma(2)} \otimes \ldots \otimes e^{\sigma(n)} \in\left(V^{*}\right)^{\otimes n} .
$$

To each edge labeled by $g$ we assign a tensor in $V^{*} \otimes V \simeq \operatorname{End}_{\mathcal{R}}(V)$ corresponding to $j_{G, n}(g) \in S L_{n}(\mathcal{R}) \subset M_{n}(\mathcal{R})$.

Let $D_{0}$ denote a graph $D$ decomposed into pieces. We assign to $D_{0}$ the tensor product of tensors corresponding to them. We denote this tensor by $T\left(D_{0}\right)$. Notice that $T\left(D_{0}\right) \in V^{\otimes N} \otimes\left(V^{*}\right)^{\otimes N}$, where $N=$ the number of 1-valent sources in $D_{0}=$ the number of 1-valent sinks in $D_{0}$.

Now we glue all components of $D_{0}$ together to get the graph $D$ back. Whenever we glue an end of one piece to a beginning of another piece in $D_{0}$, we make the corresponding contraction on $T\left(D_{0}\right)$. More specifically, suppose that the two free ends glued together correspond to the two underlined components:

$$
T\left(D_{0}\right) \in V \otimes \ldots \otimes \underline{V} \otimes \ldots \otimes V \otimes V^{*} \otimes \ldots \otimes \underline{V^{*}} \otimes \ldots \otimes V^{*} .
$$

By applying to this tensor space the contraction map $\underline{V} \otimes \underline{V^{*}} \rightarrow R$ (which is the evaluation map $(v, f) \rightarrow f(v))$, we send $T\left(D_{0}\right) \in V^{\otimes} \bar{N} \otimes \overline{\left(V^{*}\right)} \otimes N$ to an element of $V^{\otimes N-1} \otimes\left(V^{*}\right)^{\otimes N-1}$. By repeating this process until we get the graph $D$ back, we obtain an element of $\mathcal{R}$, if $D \in \mathcal{G}_{n}(G)$, or an element of $M_{n}(\mathcal{R})$, if $D \in \mathcal{G}_{n}^{\prime}(G)$. Notice that the above construction does not depend on the particular decomposition of $D$ into pieces. Therefore, we have defined functions

$$
\Theta: \mathcal{G}_{n}^{\prime}(G) \rightarrow M_{n}(\mathcal{R}), \quad \theta: \mathcal{G}_{n}(G) \rightarrow \mathcal{R}
$$

Lemma 4.4. Let $D$ be a graph in $\mathcal{G}_{n}(G)$ or in $\mathcal{G}_{n}^{\prime}(G)$. Let $w$ be an $n$-valent source of $D$ and $v$ an $n$-valent sink of $D$. Let $D_{\sigma}$, for $\sigma \in S_{n}$, be defined as at the beginning of Section 4. If $D \in \mathcal{G}_{n}(G)$, then $\theta(D)=\sum_{\sigma \in S_{n}} \epsilon(\sigma) \theta\left(D_{\sigma}\right)$. If $D \in \mathcal{G}_{n}^{\prime}(G)$, then $\Theta(D)=\sum_{\sigma \in S_{n}} \epsilon(\sigma) \Theta\left(D_{\sigma}\right)$.

Proof. We will prove Lemma 4.4 only for $D \in \mathcal{G}_{n}(G)$. For $D \in \mathcal{G}_{n}^{\prime}(G)$ the proof is identical.

Decompose $D$ and $D_{\sigma}$ into sources, sinks, and edges. We denote the fragment of the decomposition of $D$ composed of the source $w$ and the sink $v$ by $D^{0}$. We 
may assume that the decomposition of $D_{\sigma}$ is identical to that of $D$, except that it contains a coupon $D_{\sigma}^{0}$ instead of $D^{0}$ :

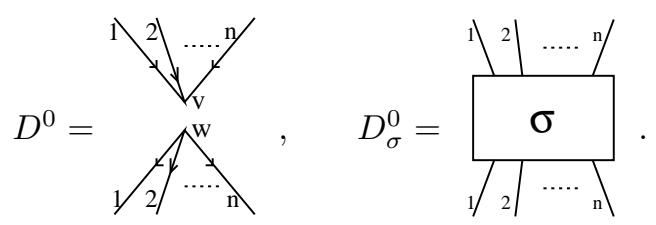

We order the 1-valent sources and sinks of $D_{\sigma}^{0}$ consistently with the ordering of the 1-valent vertices of $D^{0}$.

Let $T\left(D^{0}\right)$ (respectively, $T\left(D_{\sigma}^{0}\right)$ ) be the tensor associated to $D^{0}$ (respectively, $\left.D_{\sigma}^{0}\right)$. We assume that the $i$-th coordinate of $T\left(D^{0}\right) \in V^{\otimes n} \otimes V^{* \otimes n}$ corresponds to the $i$-th source of $D^{0}$, if $1 \leq i \leq n$, or to the $(i-n)$-th sink of $D^{0}$, if $n<i \leq 2 n$.

Recall that $\theta(D), \theta\left(D_{\sigma}\right) \in \mathcal{R}$ are results of contractions of tensors associated with elements of decompositions of $D$ and $D_{\sigma}$. Since the decompositions of $D$ and $D_{\sigma}$ chosen by us differ only by elements $D^{0}, D_{\sigma}^{0}$, the proof of Lemma 4.4 can be reduced to a local computation on tensors. Namely, it is enough to prove that

$$
T\left(D^{0}\right)=\sum_{\sigma \in S_{n}} \epsilon(\sigma) T\left(D_{\sigma}^{0}\right) .
$$

Notice that each edge of $D_{\sigma}^{0}$ is labeled by the identity map in $\operatorname{End}_{R}(V)$. This map is represented by $\sum_{i=1}^{n} e_{i} \otimes e^{i}$ in $V \otimes V^{*} \simeq \operatorname{End}_{R}(V)$. Therefore, if $\sigma=i d \in S_{n}$, then

$$
T\left(D_{\sigma}^{0}\right)=\sum_{i_{1}, i_{2}, \ldots, i_{n} \in\{1,2, \ldots, n\}} e_{i_{1}} \otimes e_{i_{2}} \otimes \ldots \otimes e_{i_{n}} \otimes e^{i_{1}} \otimes e^{i_{2}} \otimes \ldots \otimes e^{i_{n}} .
$$

Similarly, for any $\sigma \in S_{n}$, we have

$$
T\left(D_{\sigma}^{0}\right)=\sum_{i_{1}, i_{2}, \ldots, i_{n} \in\{1,2, \ldots, n\}} e_{i_{1}} \otimes e_{i_{2}} \otimes \ldots \otimes e_{i_{n}} \otimes e^{i_{\sigma(1)}} \otimes e^{i_{\sigma(2)}} \otimes \ldots \otimes e^{i_{\sigma(n)}} .
$$

Therefore,

$$
\begin{aligned}
& \sum_{\sigma \in S_{n}} \epsilon(\sigma) T\left(D_{\sigma}^{0}\right) \\
& \quad=\sum_{\substack{\sigma \in S_{n} \\
i_{1}, i_{2}, \ldots, i_{n} \in\{1,2, \ldots, n\}}} \epsilon(\sigma) e_{i_{1}} \otimes e_{i_{2}} \otimes \ldots \otimes e_{i_{n}} \otimes e^{i_{\sigma(1)}} \otimes e^{i_{\sigma(2)}} \otimes \ldots \otimes e^{i_{\sigma(n)}} .
\end{aligned}
$$

Note that we can assume that the numbers $i_{1}, i_{2}, \ldots, i_{n}$ appearing on the right side of (4.7) are all different. Indeed, if $i_{j}=i_{k}, j \neq k$, then there is an equal number of even and odd permutations contributing the term

$$
e_{i_{1}} \otimes e_{i_{2}} \otimes \ldots \otimes e_{i_{n}} \otimes e^{j_{1}} \otimes e^{j_{2}} \otimes \ldots \otimes e^{j_{n}}
$$

to the sum on the right side of (4.7), for any $j_{1}, j_{2}, \ldots, j_{n}$.

Therefore, we can assume that the numbers $\left(i_{1}, i_{2}, \ldots, i_{n}\right)$ appearing in each term of the sum on the right side of (4.7) form a permutation $\tau$ of $(1,2, \ldots, n)$. Hence we 
have

$$
\begin{aligned}
\sum_{\sigma \in S_{n}} & \epsilon(\sigma) T\left(D_{\sigma}^{0}\right) \\
& =\sum_{\sigma, \tau \in S_{n}} \epsilon(\sigma) e_{\tau(1)} \otimes e_{\tau(2)} \otimes \ldots \otimes e_{\tau(n)} \otimes e^{\tau(\sigma(1))} \otimes e^{\tau(\sigma(2))} \otimes \ldots \otimes e^{\tau(\sigma(n))} .
\end{aligned}
$$

Substitute $\tau^{\prime}$ for $\tau \circ \sigma$. Then $\epsilon(\sigma)=\epsilon(\tau) \epsilon\left(\tau^{\prime}\right)$, and we get

$$
\begin{aligned}
\sum_{\sigma \in S_{n}} & \epsilon(\sigma) T\left(D_{\sigma}^{0}\right) \\
& =\sum_{\tau, \tau^{\prime} \in S_{n}} \epsilon(\tau) \epsilon\left(\tau^{\prime}\right) e_{\tau(1)} \otimes e_{\tau(2)} \otimes \ldots \otimes e_{\tau(n)} \otimes e^{\tau^{\prime}(1)} \otimes e^{\tau^{\prime}(2)} \otimes \ldots \otimes e^{\tau^{\prime}(n)} .
\end{aligned}
$$

Notice that the right side of the above equation is equal to

$$
\left(\sum_{\tau \in S_{n}} \epsilon(\tau) e_{\tau(1)} \otimes e_{\tau(2)} \otimes \ldots \otimes e_{\tau(n)}\right) \otimes\left(\sum_{\tau^{\prime} \in S_{n}} \epsilon\left(\tau^{\prime}\right) e^{\tau^{\prime}(1)} \otimes e^{\tau^{\prime}(2)} \otimes \ldots \otimes e^{\tau^{\prime}(n)}\right) .
$$

But the expression above is exactly the tensor assigned to:

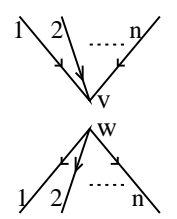

Therefore we have proved (4.6) and completed the proof of Lemma 4.4.

The study of $S L_{n}$-actions on linear spaces was one of the main objectives of classical invariant theory. In particular, Weyl (We ) determined all invariants of the action of $S L(V)$ on $V \otimes \ldots \otimes V \otimes V^{*} \otimes \ldots \otimes V^{*}$ and gave a full description of relations between them. The set of "typical" invariants consists of brackets $\left[v_{1}, \ldots, v_{n}\right]=$ $\operatorname{Det}\left(v_{1}, \ldots, v_{n}\right),\left[\phi_{1}, \ldots, \phi_{n}\right]^{*}=\operatorname{Det}\left(\phi_{1}, \ldots, \phi_{n}\right)$, where $v_{1}, \ldots, v_{n} \in V, \phi_{1}, \ldots, \phi_{n} \in V^{*}$, and contractions $\phi_{j}\left(v_{i}\right)$. The identity

$$
\left[v_{1}, \ldots, v_{n}\right]\left[\phi_{1}, \ldots, \phi_{n}\right]^{*}=\operatorname{Det}\left(\phi_{j}\left(v_{i}\right)\right)_{i, j=1}^{n}
$$

is one of the fundamental identities relating the typical invariants. The bracket $[\cdot, \cdot, \ldots, \cdot]$ is a skew symmetric linear functional on $V \otimes V \otimes \ldots \otimes V$ and hence an element of $\bigwedge^{n} V^{*}$. Similarly, $[\cdot, \cdot, \ldots, \cdot]^{*} \in \bigwedge^{n} V$. Note that the sources and sinks of graphs considered by us are labeled exactly by the tensors $[\cdot, \cdot, \ldots, \cdot]^{*}$ and $[\cdot, \cdot, \ldots, \cdot]$. (However, $V$ is in our case a free module over $\mathcal{R}=\operatorname{Rep}_{n}^{R}(G)$.)

It follows from the proof of Lemma 4.4 that

$$
\sum_{\sigma \in S_{n}} \epsilon(\sigma) \stackrel{\backslash \cdots \cdot /}{\prod|\cdots .|}
$$

represents the tensor in $H o m\left(V \otimes \ldots \otimes V \otimes V^{*} \otimes \ldots \otimes V^{*}, \mathcal{R}\right)=V^{*} \otimes \ldots \otimes V^{*} \otimes V \otimes \ldots \otimes V$ assigning to $\left(v_{1}, v_{2}, \ldots, v_{n}, \phi_{1}, \phi_{2}, \ldots, \phi_{n}\right)$ the value $\operatorname{Det}\left(\phi_{j}\left(v_{i}\right)\right)_{i, j=1}^{n}$. Therefore, the 
identity

$$
\theta(D)=\sum_{\sigma \in S_{n}} \epsilon(\sigma) \theta\left(D_{\sigma}\right)
$$

is essentially equivalent to (4.8).

Lemma 4.5. Let $L_{g}, E_{g}, E L_{g}$ be graphs defined as in Section 3 but considered as elements of $\mathcal{G}_{n}(G)$ and $\mathcal{G}_{n}^{\prime}(G)$, i.e.

(1) $L_{g} \in \mathcal{G}_{n}(G)$ is a single loop labeled by the conjugacy class of $g \in G$,

(2) $E_{g} \in \mathcal{G}_{n}^{\prime}(G)$ is a single edge labeled by $g \in G$, and

(3) $E L_{g} \in \mathcal{G}_{n}^{\prime}(G)$ is a graph composed of an edge labeled by the identity in $G$ and of a loop labeled by the conjugacy class of $g \in G$.

Under the above assumptions the functions $\Theta$ and $\theta$ satisfy conditions (1) and (2) of Theorem 3.6.

Proof. (1) $L_{g}$ can be decomposed into a single arc

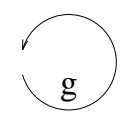

which has associated the tensor $j_{G, n}(g) \in S L_{n}(\mathcal{R}) \subset V^{*} \otimes V$. The contraction of this tensor gives $\theta\left(L_{g}\right)=\operatorname{Tr}\left(j_{G, n}(g)\right)$.

(2) $E_{g}$ is a single arc. Therefore $\Theta\left(E_{g}\right)=T\left(E_{g}\right)=j_{G, n}(g)$.

(3) $E L_{g}$ can be decomposed into:

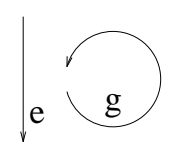

The tensor associated with this decomposition is $i d \otimes j_{G, n}(g) \in \operatorname{End}(V) \otimes$ $\operatorname{End}(V)$. After making a contraction corresponding to the identification of the ends of the arc, we get

$$
\Theta\left(E L_{g}\right)=i d \cdot \operatorname{Tr}\left(j_{G, n}(g)\right) \in \operatorname{End}(V) .
$$

Lemma 4.6. Let $D, D^{\prime} \in \mathcal{G}_{n}(G)$ be two graphs which are identical as unlabeled graphs and which have the same labeling of edges and loops except the labeling of edges incident to a vertex $v$. Moreover, suppose that

(1) if $v$ is a source, then the edges in $D$ incident to $v$ are labeled by $g_{1}, g_{2}, \ldots, g_{n}$ and the edges in $D^{\prime}$ incident to $v$ are labeled by $g_{1} h, g_{2} h, \ldots, g_{n} h$ for some $g_{1}, g_{2}, \ldots, g_{n}, h \in G$;

(2) if $v$ is a sink, then the edges in $D$ incident to $v$ are labeled by $g_{1}, g_{2}, \ldots, g_{n}$ and the edges in $D^{\prime}$ incident to $v$ are labeled by $h g_{1}, h g_{2}, \ldots, h g_{n}$ for some $g_{1}, g_{2}, \ldots, g_{n}, h \in G$.

Under the above assumptions $\theta(D)=\theta\left(D^{\prime}\right)$. An analogous fact is true for graphs in $\mathcal{G}_{n}^{\prime}(G)$.

Proof. We prove part (1) only. The proof of part (2) is analogous.

Let $v$ be a source. Notice that $D$ and $D^{\prime}$ have identical decompositions into sinks, sources, and arcs except that $D_{0}=\bigvee \ldots /$ is an element of the decomposition of 
$D$ and the diagram $D_{0}^{\prime}=$

Therefore we need to show that the tensors assigned to the above diagrams are identical. Notice that the tensor associated to $D_{0}, T\left(D_{0}\right)$, is an element of the one-dimensional $\mathcal{R}$-linear space of skew-symmetric tensors $\bigwedge^{n} V \subset V^{n}$. Let $A$ : $V \rightarrow V$ be an endomorphism given in the standard coordinates of $V$ by $j_{G, n}(h) \in$ $S L_{n}(\mathcal{R})$. $A$ induces an endomorphism $\wedge A: \bigwedge^{n} V \rightarrow \bigwedge^{n} V$ with the property that $\wedge A\left(T\left(D_{0}\right)\right)=\operatorname{Det}(A) T\left(D_{0}\right) \in \wedge^{n} V$. Notice that $\wedge A\left(T\left(D_{0}\right)\right)$ is exactly the tensor associated to $D_{0}^{\prime}$. Since $\operatorname{Det}(A)=1$, the tensors associated to $D_{0}$ and $D_{0}^{\prime}$ are equal.

Proof of Theorem 3.6. Let us extend the functions $\theta$ and $\Theta$ to all $R$-linear combinations of graphs in $\mathcal{G}_{n}(G)$ and $\mathcal{G}_{n}^{\prime}(G)$ respectively. Facts 4.1 and 4.2 and Lemmas 4.4, 4.5, and 4.6 imply that these functions descend to $R$-linear homomorphisms

$$
\Theta: \mathbb{A}_{n}\left(X, x_{0}\right) \rightarrow M_{n}(\mathcal{R}), \quad \theta: \mathbb{A}_{n}(X) \rightarrow \mathcal{R} .
$$

By Lemma 4.5 $\Theta$ and $\theta$ satisfy conditions (1) and (2) of Theorem 3.6

We have showed in Proposition 3.54) that $\mathbb{A}_{n}\left(X, x_{0}\right)$ is generated by elements $E_{\gamma}$ and $E L_{\gamma^{\prime}}$, for $\gamma, \gamma^{\prime} \in G=\pi_{1}\left(X, x_{0}\right)$. By Proposition 2.4 and the paragraph preceding it, $\Theta\left(E_{\gamma}\right)=j_{G, n}(\gamma)$ and $\Theta\left(E L_{\gamma^{\prime}}\right)=\operatorname{Tr}\left(j_{G, n}\left(\gamma^{\prime}\right)\right)$ belong to

$$
M_{n}\left(\operatorname{Rep}_{n}^{R}(G)\right)^{G L_{n}(R)} .
$$

Therefore the image of $\Theta$ lies in $M_{n}\left(\operatorname{Rep}_{n}^{R}(G)\right)^{G L_{n}(R)}$. We show analogously that the image of $\theta$ lies in $\operatorname{Rep}_{n}^{R}(G)^{G L_{n}(R)}$. Therefore the proof will be completed if we show that the diagram of Theorem 3.6 commutes.

Let $D \in \mathcal{G}_{n}^{\prime}(G), G=\pi_{1}\left(X, x_{0}\right)$, represent an element of $\mathbb{A}_{n}\left(X, x_{0}\right)$. Then $\Theta(D) \in M_{n}\left(\operatorname{Rep}_{n}^{R}(G)\right)^{G L_{n}(R)}$ is the result of a contraction of tensors associated with elements of some decomposition of $D$. Notice that $\mathbb{T}(D)$ is an element of $\mathbb{A}_{n}(X)$ represented by the diagram $D$ with its 1 -valent vertices identified 2 Hence $\theta(\mathbb{T}(D))$ is a contraction of $\Theta(D)$, i.e. $\theta(\mathbb{T}(D))=\operatorname{Tr}(\Theta(D))$. Since the elements $D \in \mathcal{G}_{n}^{\prime}(G)$ span $\mathbb{A}_{n}\left(X, x_{0}\right)$, the diagram of Theorem 3.6 commutes.

\section{Proof of Theorem 3.7}

Now we assume that $R$ is a field of characteristic 0 .

We start by stating the first and second fundamental theorems of invariant theory, following the approach of Procesi, Pro-1] (compare also [Ra]).

Let $I$ be an infinite set. Let $P_{n}(I)$ and $A_{i}$ be as before,

$$
P_{n}(I)=R\left[x_{j k}^{i}, j, k \in\{1,2, \ldots, n\}, i \in I\right], \quad A_{i}=\left(x_{j k}^{i}\right) \in M_{n}\left(P_{n}(I)\right) .
$$

We are going to present Procesi's description of the ring $M_{n}\left(P_{n}(I)\right)^{G L_{n}(R)}$.

Let $T$ be a commutative $R$-algebra freely generated by the symbols

$$
\operatorname{Tr}\left(X_{i_{1}} X_{i_{2}} \ldots X_{i_{k}}\right),
$$

where $i_{1}, i_{2}, \ldots, i_{k} \in I$. We adopt the convention that $\operatorname{Tr}(M)=\operatorname{Tr}(N)$ if and only if the monomial $N$ is obtained from $M$ by a cyclic permutation. Let $T\left\{X_{i}\right\}_{i \in I}$ be a non-commutative $T$-algebra freely generated by variables $X_{i}, i \in I$. We

\footnotetext{
${ }^{2}$ Recall that $\mathbb{T}$ was defined in the paragraph preceding Fact 3.4
} 
have a natural $T$-linear homomorphism $T r: T\left\{X_{i}\right\}_{i \in I} \rightarrow T$ which assigns to $X_{i_{1}} X_{i_{2}} \ldots X_{i_{k}} \in T\left\{X_{i}\right\}_{i \in I}$ an element $\operatorname{Tr}\left(X_{i_{1}} X_{i_{2}} \ldots X_{i_{k}}\right) \in T$.

There is a homomorphism $\pi: T\left\{X_{i}\right\}_{i \in I} \rightarrow M_{n}\left(P_{n}(I)\right)$ uniquely determined by the conditions:

- $\pi\left(X_{i}\right)=A_{i}$

- $\pi\left(\operatorname{Tr}\left(X_{i_{1}} X_{i_{2}} \ldots X_{i_{k}}\right)\right)=\operatorname{Tr}\left(A_{i_{1}} A_{i_{2}} \ldots A_{i_{k}}\right) \in P_{n}(I) \subset M_{n}\left(P_{n}(I)\right) 3$

Proposition 2.3 and Lemma 2.2 imply that the image of $\pi$ is fixed by the $G L_{n}(R)$ action on $M_{n}\left(P_{n}(I)\right)$, i.e. $\pi: T\left\{X_{i}\right\}_{i \in I} \rightarrow M_{n}\left(P_{n}(I)\right)^{G L_{n}(R)}$.

Notice that the following diagram commutes:

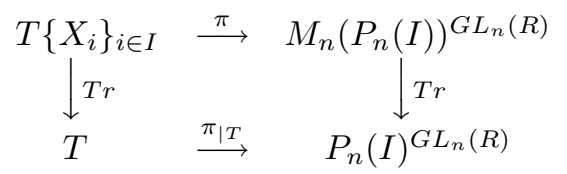

The following version of The First Fundamental Theorem of invariant theory of $n \times n$ matrices is due to Procesi, Pro-1].

Theorem 5.1. $\pi: T\left\{X_{i}\right\}_{i \in I} \rightarrow M_{n}\left(P_{n}(I)\right)^{G L_{n}(R)}$ is an epimorphism.

Before we state the second fundamental theorem of invariant theory of $n \times n$ matrices, we need some preparations.

Suppose that $\{1,2, \ldots, m\} \subset I$ and specify $i_{0} \in\{1,2, \ldots, m\}$. We can present any $\sigma \in S_{m}$ as a product of cycles in such a way that $i_{0}$ is the first element of the first cycle, $\sigma=\left(i_{0}, i_{1}, \ldots, i_{s}\right)\left(j_{0}, j_{1}, \ldots, j_{t}\right) \ldots\left(k_{0}, k_{1}, \ldots, k_{v}\right)$. We define $\Phi_{\sigma, i_{0}}\left(X_{1}, X_{2}, \ldots, X_{m}\right)$ to be equal to

$$
X_{i_{0}} X_{i_{1}} \ldots X_{i_{s}} \operatorname{Tr}\left(X_{j_{0}} X_{j_{1}} \ldots X_{j_{t}}\right) \ldots \operatorname{Tr}\left(X_{k_{0}} X_{k_{1}} \ldots X_{k_{v}}\right) \in T\left\{X_{i}\right\}_{i \in I} .
$$

We also define another expression which does not depend on $i_{0}$ :

$\Phi_{\sigma}\left(X_{1}, X_{2}, \ldots, X_{m}\right)=\operatorname{Tr}\left(X_{i_{0}} X_{i_{1}} \ldots X_{i_{s}}\right) \operatorname{Tr}\left(X_{j_{0}} X_{j_{1}} \ldots X_{j_{t}}\right) \ldots \operatorname{Tr}\left(X_{k_{0}} X_{k_{1}} \ldots X_{k_{v}}\right) \in T$.

Let

$$
F\left(X_{1}, X_{2}, \ldots, X_{m}\right)=\sum_{\sigma \in S_{m}} \epsilon(\sigma) \Phi_{\sigma}\left(X_{1}, X_{2}, \ldots, X_{m}\right) \in T .
$$

$F\left(X_{1}, X_{2}, \ldots, X_{n+1}\right)$ is called the fundamental trace identity of $n \times n$ matrices.

Procesi argues that there exists a unique element $G\left(X_{1}, X_{2}, \ldots, X_{n}\right) \in T\left\{X_{i}\right\}_{i \in I}$, involving only the variables $X_{1}, \ldots, X_{n}$ and the traces of monomials in these variables, such that

$$
F\left(X_{1}, X_{2}, \ldots, X_{n+1}\right)=\operatorname{Tr}\left(G\left(X_{1}, X_{2}, \ldots, X_{n}\right) X_{n+1}\right) \in T\left\{X_{i}\right\}_{i \in I} .
$$

Procesi gives an explicit formula for $G\left(X_{1}, X_{2}, \ldots, X_{n}\right)$, but we want to give a different formula, which will be more suitable for our purposes.

\section{Lemma 5.2.}

$$
\begin{aligned}
& G\left(X_{1}, X_{2}, \ldots, X_{n}\right) \\
& \quad=\sum_{\sigma \in S_{n}} \epsilon(\sigma) \Phi_{\sigma}\left(X_{1}, X_{2}, \ldots, X_{n}\right)-\sum_{\substack{i \in\{1,2, \ldots, n\} \\
\sigma \in S_{n}}} \epsilon(\sigma) \Phi_{\sigma, i}\left(X_{1}, X_{2}, \ldots, X_{n}\right) .
\end{aligned}
$$

\footnotetext{
${ }^{3}$ We identify $P_{n}(I)$ with the scalar matrices in $M_{n}\left(P_{n}(I)\right)$.
} 
Proof. It follows from the remarks preceding Lemma 5.2 that it is enough to show that if we multiply the right side of the equation of Lemma [5.2 by $X_{n+1}$, then the trace of it will be equal to $F\left(X_{1}, \ldots, X_{n+1}\right)$, i.e. we have to prove that

$$
\begin{gathered}
\sum_{\sigma \in S_{n}} \epsilon(\sigma) \Phi_{\sigma}\left(X_{1}, X_{2}, \ldots, X_{n}\right) \operatorname{Tr}\left(X_{n+1}\right)-\sum_{\substack{i \in\{, 2, \ldots, n\} \\
\sigma \in S_{n}}} \epsilon(\sigma) \operatorname{Tr}\left(\Phi_{\sigma, i}\left(X_{1}, X_{2}, \ldots, X_{n}\right) X_{n+1}\right) \\
=F\left(X_{1}, X_{2}, \ldots, X_{n+1}\right) .
\end{gathered}
$$

Notice that $\epsilon(\sigma) \Phi_{\sigma}\left(X_{1}, X_{2}, \ldots, X_{n}\right) \operatorname{Tr}\left(X_{n+1}\right)=\epsilon\left(\sigma^{\prime}\right) \Phi_{\sigma^{\prime}}\left(X_{1}, X_{2}, \ldots, X_{n}, X_{n+1}\right)$, where $\sigma^{\prime} \in S_{n+1}, \sigma^{\prime}(i)=\sigma(i)$, for $i \in\{1,2, \ldots, n\}$, and $\sigma^{\prime}(n+1)=n+1$.

Similarly we can simplify $\operatorname{Tr}\left(\Phi_{\sigma, i}\left(X_{1}, X_{2}, \ldots, X_{n}\right) X_{n+1}\right)$. Suppose that

$$
\sigma=\left(i_{0}, i_{1}, \ldots, i_{s}\right)\left(j_{0}, j_{1}, \ldots, j_{t}\right) \ldots\left(k_{0}, k_{1}, \ldots, k_{v}\right) \in S_{n},
$$

where $i_{0}=i$. Then

$$
\operatorname{Tr}\left(\Phi_{\sigma, i}\left(X_{1}, X_{2}, \ldots, X_{n}\right) X_{n+1}\right)=\Phi_{\sigma^{\prime}}\left(X_{1}, X_{2}, \ldots, X_{n}, X_{n+1}\right),
$$

for $\sigma^{\prime}=\left(i_{0}, i_{1}, \ldots, i_{s}, n+1\right)\left(j_{0}, j_{1}, \ldots, j_{t}\right) \ldots\left(k_{0}, k_{1}, \ldots, k_{v}\right) \in S_{n+1}$. Notice that $\epsilon\left(\sigma^{\prime}\right)=$ $-\epsilon(\sigma)$. Therefore the left side of the equation (5.1) is equal to

$$
\begin{aligned}
& \sum_{\substack{\sigma^{\prime} \in S_{n+1}, \sigma^{\prime}(n+1)=n+1}} \epsilon\left(\sigma^{\prime}\right) \Phi_{\sigma}\left(X_{1}, X_{2}, \ldots, X_{n}, X_{n+1}\right) \\
& +\sum_{\substack{i \in\{1,2, \ldots, n\}, \sigma^{\prime} \in S_{n+1} \\
\text { such that } \sigma^{\prime}(n+1)=i}} \epsilon\left(\sigma^{\prime}\right) \Phi_{\sigma}\left(X_{1}, X_{2}, \ldots, X_{n}, X_{n+1}\right) .
\end{aligned}
$$

The above expression is obviously equal to $F\left(X_{1}, X_{2}, \ldots, X_{n+1}\right)$.

Now we are ready to state The Second Fundamental Theorem of invariant theory of $n \times n$ matrices, Pro-1.

Theorem 5.3. The kernel of $\pi$ is generated by elements $G\left(M_{1}, M_{2}, \ldots, M_{n}\right)$ and $F\left(N_{1}, N_{2}, \ldots, N_{n+1}\right)$, where $M_{1}, M_{2}, \ldots, M_{n}, N_{1}, N_{2}, \ldots, N_{n+1}$ are all possible monomials in the variables $X_{i}, i \in I$.

Let $X$ be a path-connected topological space. We choose a presentation $\left\langle g_{i}, i \in\right.$ $I\left|r_{j}, j \in J\right\rangle$ of $G=\pi_{1}\left(X, x_{0}\right)$ such that

- $I$ is an infinite set,

- the inverse of every generator is also a generator, and

- the defining relations $r_{j}$ are products of non-negative powers of generators.

Note that such a presentation always exists (even if $G$ is finitely generated).

Let $\psi: T\left\{X_{i}\right\}_{i \in I} \rightarrow \mathbb{A}_{n}\left(X, x_{0}\right)$ be an $R$-homomorphism such that $\psi\left(X_{i}\right)=E_{g_{i}}$ and $\psi\left(\operatorname{Tr}\left(X_{i_{1}} X_{i_{2}} \ldots X_{i_{k}}\right)\right)=E L_{g_{i_{1}} g_{i_{2}} \ldots g_{i_{k}}}$. Recall that, by Proposition 3.4. $\mathbb{A}_{n}(X)$ can be considered as a subalgebra of $\mathbb{A}_{n}\left(X, x_{0}\right)$ in such a way that $L_{\gamma} \in \mathbb{A}_{n}(X)$ is identified with $E L_{\gamma} \in \mathbb{A}_{n}\left(X, x_{0}\right)$. Hence $\psi\left(\operatorname{Tr}\left(X_{i_{1}} X_{i_{2}} \ldots X_{i_{k}}\right)\right) \in \mathbb{A}_{n}(X)$ and $\psi$ restricts to $\psi: T \rightarrow \mathbb{A}_{n}(X)$. Moreover, the following diagram commutes:

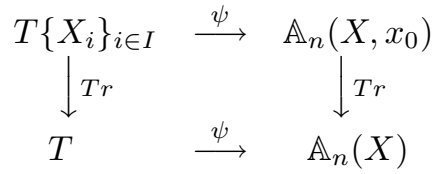


We are going to show that the kernel of $\psi: T\left\{X_{i}\right\}_{i \in I} \rightarrow \mathbb{A}_{n}\left(X, x_{0}\right)$ contains the kernel of $\pi: T\left\{X_{i}\right\}_{i \in I} \rightarrow M_{n}\left(P_{n}(I)\right)^{G L_{n}(R)}$ and therefore $\psi$ descends to a homomorphism $M_{n}\left(P_{n}(I)\right)^{G L_{n}(R)} \rightarrow \mathbb{A}_{n}\left(X, x_{0}\right)$.

We will need the following fact, due to Formanek (Proposition 45 For $)$.

Proposition 5.4. For any matrix $A \in M_{n}(R)$

$$
\operatorname{Det}(A)=\frac{1}{n !} \sum_{\sigma \in S_{n}} \epsilon(\sigma) \operatorname{Tr}\left(A^{c_{1}}\right) \operatorname{Tr}\left(A^{c_{2}}\right) \ldots \operatorname{Tr}\left(A^{c_{k}}\right),
$$

where $c_{1}, c_{2}, \ldots, c_{k}$ denote the lengths of all cycles in $\sigma$.

For completeness we sketch a proof of Proposition [5.4. A multilinearization of the determinant, Det $: M_{n}(R) \rightarrow R$, gives a function on $n$-tuples of $n \times n$ matrices,

$$
\mathcal{M}\left(X_{1}, \ldots, X_{n}\right)=\sum_{\sigma \in S_{n}} \operatorname{Det}\left(X_{\sigma}\right)
$$

where $X_{\sigma}$ is a matrix whose $i$-th row is the $i$-th row of $X_{\sigma(i)}$. Note that $\mathcal{M}(A, \ldots, A)$ $=n ! \operatorname{Det}(A)$, and therefore the identity of Proposition 5.4 is a special case of the following identity:

$$
\mathcal{M}\left(X_{1}, \ldots, X_{n}\right)=\sum_{\sigma \in S_{n}} \epsilon(\sigma) \Phi_{\sigma}\left(X_{1}, \ldots, X_{n}\right),
$$

where $\Phi_{\sigma}$ was defined in the second paragraph after Theorem 5.1 Formanek gives the following proof of the above identity. Assume that $1,2, \ldots, n \in I$. Since $A_{1}, \ldots, A_{n} \in M_{n}\left(P_{n}(I)\right)$ represent generic matrices, in order to prove the above identity it is enough to show it for $X_{1}=A_{1}, \ldots, X_{n}=A_{n}$. Since $\mathcal{M}\left(A_{1}, \ldots, A_{n}\right)$ is an invariant polynomial function on $n$-tuples of matrices, the First Fundamental Theorem implies that $\mathcal{M}\left(A_{1}, \ldots, A_{n}\right)$ can be expressed in terms of traces of monomials in $A_{1}, \ldots, A_{n}$. Since $\mathcal{M}\left(A_{1}, \ldots, A_{n}\right)$ is linear with respect to $A_{1}, \ldots, A_{n}$, it is a linear combination of terms $\operatorname{Tr}\left(A_{i_{1}} \ldots A_{i_{s}}\right) \ldots \operatorname{Tr}\left(A_{j_{1}} \ldots A_{j_{t}}\right)$, where $i_{1}, \ldots i_{s}, \ldots, j_{1}, \ldots, j_{t}$ form a permutation of $1,2, \ldots, n$. Therefore

$$
\mathcal{M}\left(A_{1}, \ldots, A_{n}\right)=\sum_{\sigma \in S_{n}} \alpha_{\sigma} \Phi_{\sigma}\left(A_{1}, \ldots, A_{n}\right),
$$

and hence

$$
\mathcal{M}\left(X_{1}, \ldots, X_{n}\right)=\sum_{\sigma \in S_{n}} \alpha_{\sigma} \Phi_{\sigma}\left(X_{1}, \ldots, X_{n}\right),
$$

for any $n \times n$ matrices $X_{1}, \ldots, X_{n}$. We need to prove that $\alpha_{\sigma}=\epsilon(\sigma)$. If we restrict the above equation to matrices $A_{1}, \ldots, A_{n} \in M_{n-1}\left(P_{n-1}(I)\right)$ embedded into $M_{n}\left(P_{n-1}(I)\right)$ in the standard, non-unit-preserving way, we will get the following polynomial identity on $(n-1) \times(n-1)$ matrices:

$$
\sum_{\sigma \in S_{n}} \alpha_{\sigma} \Phi\left(A_{1}, \ldots, A_{n}\right)=0 .
$$

It is not difficult to see that the Second Fundamental Theorem implies that $F\left(A_{1}, \ldots, A_{n}\right)$ is the only (up to scalar) $n$-linear trace identity of degree $n$ on matrices $A_{1}, \ldots, A_{n} \in M_{n-1}\left(P_{n-1}(I)\right)$. Therefore $\alpha_{\sigma}=\epsilon(\sigma) c$, for some fixed $c$. Substituting the matrix $\left(x_{i j}\right)$ with a single non-zero entry $x_{i i}=1$ for $X_{i}$ in (5.3), we get $c=1$. Thus the proof of Proposition 5.4 is finished.

The specialization $A=I d \in M_{n}(R)$ in Proposition 5.4 yields the following corollary. 
Corollary 5.5. For any positive integer $n$,

$$
\sum_{\sigma \in S_{n}} \epsilon(\sigma) n^{c(\sigma)}=n !
$$

where $c(\sigma)$ is the number of cycles in the cycle decomposition of $\sigma$.

From the definition of $T\left\{X_{i}\right\}_{i \in I}$ it immediately follows that for any family of matrices $\left\{M_{i}\right\}_{i \in I} \subset M_{n}(R)$ there is a well-defined substitution

$$
X_{i} \rightarrow M_{i}, \quad \operatorname{Tr}\left(X_{i_{1}} X_{i_{2}} \ldots X_{i_{k}}\right) \rightarrow \operatorname{Tr}\left(M_{i_{1}} M_{i_{2}} \ldots M_{i_{k}}\right) \in R \subset M_{n}(R),
$$

which can be extended to the whole ring $T\left\{X_{i}\right\}_{i \in I}$. Therefore, if $H\left(X_{i_{1}}, \ldots, X_{i_{k}}\right)$ is an element of $T\left\{X_{i}\right\}_{i \in I}$ involving variables $X_{i_{1}}, \ldots, X_{i_{k}}$, then $H\left(M_{i_{1}}, \ldots, M_{i_{k}}\right)$ is a well-defined matrix in $M_{n}(R)$.

Lemma 5.6. If $N_{1}, N_{2}, \ldots, N_{n}$ are any monomials in the variables $X_{i}, i \in I$, then $\psi\left(G\left(N_{1}, N_{2}, \ldots, N_{n}\right)\right)=0$.

Proof. By the definition of $\psi$ (given in the second paragraph after Theorem 5.3), $\psi\left(N_{i}\right)=E_{h_{i}}$, for some $h_{1}, h_{2}, \ldots, h_{n} \in G$. Consider the following graph $D$ in $\mathcal{G}_{n}^{\prime}(G)$ :
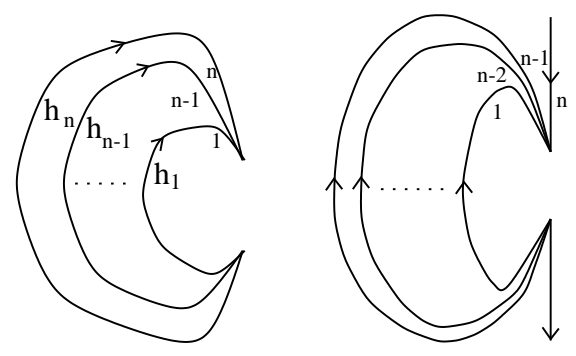

in which we omitted labels of edges labeled by the identity in $G$. Notice that $D$ can also be presented in the following way:

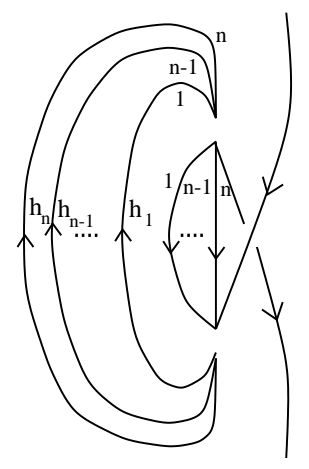

Since the vertices of $D$ can be resolved in two possible ways (corresponding to the two diagrams above), we obtain the following equation:

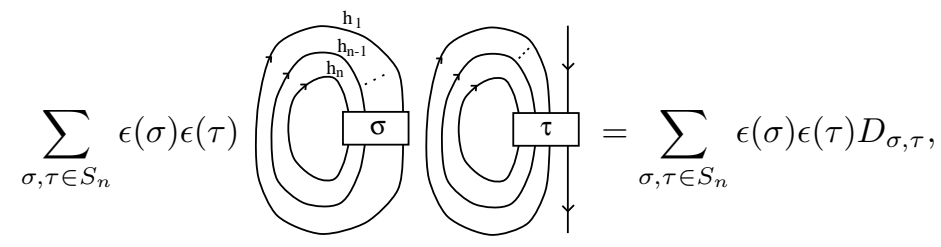


where $D_{\sigma, \tau}$ is a graph of the form:

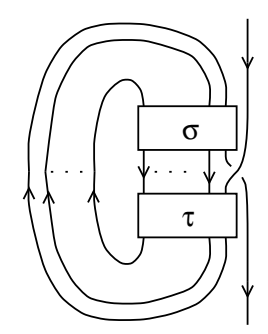

If $\tau \in S_{n}$ decomposes into $k=c(\tau)$ cycles, then

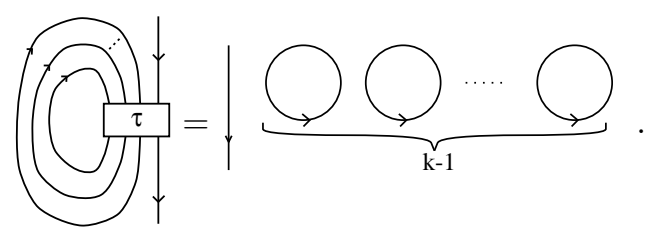

Therefore, by Corollary [5.5,

$$
\sum_{\tau \in S_{n}} \epsilon(\tau)=\sum_{\tau \in S_{n}} \epsilon(\tau) n^{c(\tau)-1}=(n-1) !
$$

Notice moreover that

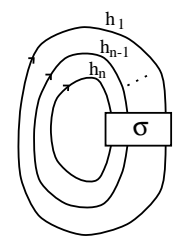

is equal to $\psi\left(\Phi_{\sigma}\left(N_{1}, N_{2}, \ldots, N_{n}\right)\right)$. Therefore the left side of (5.4) is equal to the value of $\psi$ on

$$
(n-1) ! \sum_{\sigma \in S_{n}} \epsilon(\sigma) \Phi_{\sigma}\left(N_{1}, N_{2}, \ldots, N_{n}\right) .
$$

Now we are going to consider the right side of (5.4). Notice that the single arc in $D_{\sigma, \tau}$ is labeled by an element $h_{i_{s}} \ldots h_{i_{1}} h_{i_{0}} \in G$, where $i_{0}=\tau(n), i_{1}=\tau \sigma\left(i_{0}\right), \ldots, i_{s}=$ $\tau \sigma\left(i_{s-1}\right)$, and $\sigma\left(i_{s}\right)=n$. Since $\tau\left(\sigma\left(i_{s}\right)\right)=\tau(n)=i_{0},\left(i_{s}, i_{s-1}, \ldots, i_{1}, i_{0}\right)$ is a cycle of the permutation $(\tau \sigma)^{-1} \in S_{n}$.

Note that every loop in $D_{\sigma, \tau}$ is labeled by the conjugacy class of $h_{j_{t}} h_{j_{t-1}} \ldots h_{j_{1}} h_{j_{0}}$, where $\left(j_{t}, j_{t-1}, \ldots, j_{1}, j_{0}\right)$ is a cycle of $(\tau \sigma)^{-1} \in S_{n}$ disjoint from $\left(i_{s}, i_{s-1}, \ldots, i_{1}, i_{0}\right)$. Therefore $D_{\sigma, \tau}$ is the value of $\psi$ on

$$
N_{i_{s}} \ldots N_{i_{1}} N_{i_{0}} \operatorname{Tr}\left(N_{j_{t}} \ldots N_{j_{1}} N_{j_{0}}\right) \ldots \operatorname{Tr}\left(N_{k_{v}} \ldots N_{k_{1}} N_{k_{0}}\right)
$$

where

$$
\left(i_{s}, \ldots, i_{1}, i_{0}\right)\left(j_{t}, \ldots, j_{1}, j_{0}\right) \ldots\left(k_{v}, \ldots, k_{1}, k_{0}\right)
$$


is the cycle decomposition of $(\tau \sigma)^{-1}$. The above expression is equal to

$$
\Phi_{(\tau \sigma)^{-1}, i_{s}}\left(N_{1}, N_{2}, \ldots, N_{n}\right)=\Phi_{(\tau \sigma)^{-1}, \sigma^{-1}(n)}\left(N_{1}, N_{2}, \ldots, N_{n}\right) .
$$

Therefore, the right side of (5.4) is the value of $\psi$ on

$$
\sum_{\sigma, \tau \in S_{n}} \epsilon(\sigma) \epsilon(\tau) \Phi_{(\tau \sigma)^{-1}, \sigma^{-1}(n)}\left(N_{1}, N_{2}, \ldots, N_{n}\right) \in T\left\{X_{i}\right\}_{i \in I} .
$$

Let us replace $(\tau \sigma)^{-1}$ by $\kappa$ in the expression above. Then we get

$$
\sum_{\sigma, \kappa \in S_{n}} \epsilon(\kappa) \Phi_{\kappa, \sigma^{-1}(n)}\left(N_{1}, N_{2}, \ldots, N_{n}\right)=(n-1) ! \sum_{\kappa \in S_{n},} \epsilon(\kappa) \Phi_{\kappa, i}\left(N_{1}, N_{2}, \ldots, N_{n}\right) .
$$

After comparing the above algebraic descriptions of the two sides of (5.4) we see that for any monomials $N_{1}, N_{2}, \ldots, N_{n}$ the following element of $T\left\{X_{i}\right\}_{i \in I}$ belongs to $\operatorname{Ker} \psi$ :

$$
\sum_{\sigma \in S_{n}} \epsilon(\sigma) \Phi_{\sigma}\left(N_{1}, N_{2}, \ldots, N_{n}\right)-\sum_{\kappa \in S_{n},} \sum_{i \in\{1,2, \ldots, n\}} \epsilon(\kappa) \Phi_{\kappa, i}\left(N_{1}, N_{2}, \ldots, N_{n}\right) .
$$

By Lemma 5.2 the above expression is equal to $G\left(N_{1}, N_{2}, \ldots, N_{n}\right)$. Therefore $\psi\left(G\left(N_{1}, N_{2}, \ldots, N_{n}\right)\right)=0$.

Lemma 5.7. Let $N_{1}, N_{2}, \ldots, N_{n+1}$ be any monomials in the variables $X_{i}, i \in I$. Then $\psi\left(F\left(N_{1}, N_{2}, \ldots, N_{n+1}\right)\right)=0$.

Proof. By definition, $F\left(N_{1}, N_{2}, \ldots, N_{n+1}\right)=\operatorname{Tr}\left(G\left(N_{1}, N_{2}, \ldots, N_{n}\right) N_{n+1}\right)$. By (5.2), $\psi$ commutes with the trace function. Therefore

$$
\begin{gathered}
\psi\left(F\left(N_{1}, N_{2}, \ldots, N_{n+1}\right)\right)=\psi\left(\operatorname{Tr}\left(G\left(N_{1}, N_{2}, \ldots, N_{n}\right) N_{n+1}\right)\right) \\
=\operatorname{Tr}\left(\psi\left(G\left(N_{1}, N_{2}, \ldots, N_{n}\right)\right) \psi\left(N_{n+1}\right)\right)=0 .
\end{gathered}
$$

Lemmas 5.6 and 5.7 and the Second Fundamental Theorem imply that the kernel of $\psi: T\left\{X_{i}\right\}_{i \in I} \rightarrow \mathbb{A}_{n}\left(X, x_{0}\right)$ contains the kernel of $\pi: T\left\{X_{i}\right\}_{i \in I} \rightarrow$ $M_{n}\left(P_{n}(I)\right)^{G L_{n}(R)}$. Therefore we have the following corollary.

Corollary 5.8. There exists an R-algebra homomorphism $\psi^{\prime}: M_{n}\left(P_{n}(I)\right)^{G L_{n}(R)}$ $\rightarrow \mathbb{A}_{n}\left(X, x_{0}\right)$ such that $\psi^{\prime}\left(A_{i}\right)=E_{g_{i}}$ and $\psi^{\prime}\left(\operatorname{Tr}\left(A_{i_{1}} A_{i_{2}} \ldots A_{i_{k}}\right)\right)=E L_{g_{i_{1}} g_{i_{2}} \ldots g_{i_{k}}}$, for any $i_{1}, i_{2}, \ldots, i_{k} \in I$.

The epimorphism $\eta: P_{n}(I) \rightarrow \operatorname{Rep}_{n}^{R}(G)$ introduced in Section 2 induces an epimorphism $M_{n}(\eta): M_{n}\left(P_{n}(I)\right) \rightarrow M_{n}\left(\operatorname{Rep} p_{n}^{R}(G)\right)$ and, therefore, by restriction, a homomorphism $M_{n}(\eta)^{G L_{n}(R)}: M_{n}\left(P_{n}(I)\right)^{G L_{n}(R)} \rightarrow M_{n}\left(\operatorname{Rep} p_{n}^{R}(G)\right)^{G L_{n}(R)}$. Our goal is to show that $\psi^{\prime}$ descends to

$$
\psi^{\prime \prime}: M_{n}\left(\operatorname{Rep}_{n}^{R}(G)\right)^{G L_{n}(R)} \rightarrow \mathbb{A}_{n}\left(X, x_{0}\right)
$$

such that the following diagram commutes:

$$
\begin{array}{cl}
M_{n}\left(P_{n}(I)\right)^{G L_{n}(R)} & \\
\downarrow M_{n}(\eta)^{G L_{n}(R)} & \mho^{\psi^{\prime}} \\
M_{n}\left(\operatorname{Rep}_{n}^{R}(G)\right)^{G L_{n}(R)} \stackrel{\psi^{\prime \prime}}{\longrightarrow} & \mathbb{A}_{n}\left(X, x_{0}\right)
\end{array}
$$


In order to prove this fact we need to show that $\operatorname{Ker} M_{n}(\eta)^{G L_{n}(R)} \subset \operatorname{Ker} \psi^{\prime}$. We will use the following lemma.

Lemma 5.9. (1) $\operatorname{Det}\left(A_{i}\right) \in P_{n}(I)^{G L_{n}(R)} \subset M_{n}\left(P_{n}(I)\right)^{G L_{n}(R)}$.

(2) $\psi^{\prime}\left(\operatorname{Det}\left(A_{i}\right)\right)=1$, for any $i \in I$.

Proof. (1) By Proposition $5.4 \operatorname{Det}\left(A_{i}\right)$ can be expressed as a linear combination of traces of powers of $A_{i}$. By Lemma $2.2(2), A_{i}^{k} \in M_{n}\left(P_{n}(I)\right)^{G L_{n}(R)}$, and hence, by Proposition [2.3] $\operatorname{Tr}\left(A_{i}^{k}\right) \in P_{n}(I)^{G L_{n}(R)}$. Finally, by Lemma [2.2(1) there is a natural embedding $P_{n}(I)^{G L_{n}(R)} \subset M_{n}\left(P_{n}(I)\right)^{G L_{n}(R)}$.

(2) If $c_{1}, c_{2}, \ldots, c_{k}$ are the lengths of all cycles of $\sigma \in S_{n}$, then $\psi^{\prime}$ maps

$$
\operatorname{Tr}\left(A_{i}^{c_{1}}\right) \operatorname{Tr}\left(A_{i}^{c_{2}}\right) \ldots \operatorname{Tr}\left(A_{i}^{c_{k}}\right)
$$

to a graph

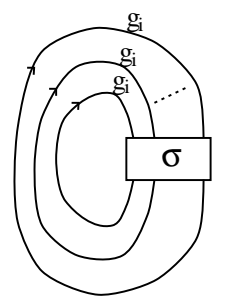

Therefore, by Proposition 5.4 and Fact 4.1 ,

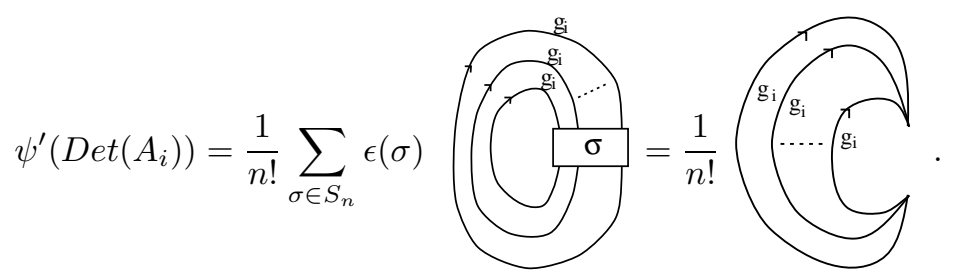

Analogously,

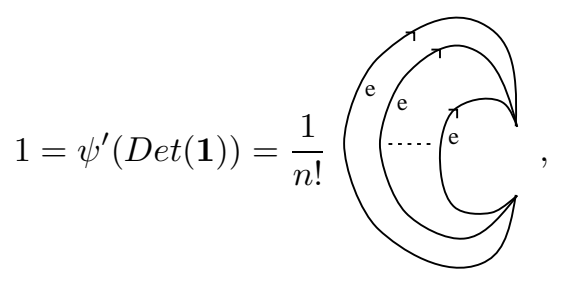

where $e$ is the identity in $G$. But, by (4.3) (or, equivalently, (4.4)),

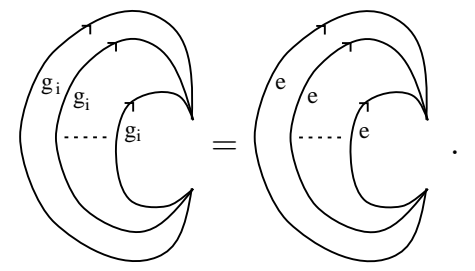

Therefore $\psi^{\prime}\left(\operatorname{Det}\left(A_{i}\right)\right)=1$. 
The next proposition is due to Procesi. Since the proof of this proposition is hidden in the proof of Theorem 2.6 in [Pro-2], we will recall it here for completeness of this paper 4

Proposition 5.10. Let $\mathcal{J} \triangleleft M_{n}\left(P_{n}(I)\right)^{G L_{n}(R)}$ be a two-sided ideal and let $\mathcal{J}^{\prime}$ be the ideal in $P_{n}(I)$ generated by the entries of elements of $M_{n}\left(P_{n}(I)\right) \mathcal{J} M_{n}\left(P_{n}(I)\right) \triangleleft$ $M_{n}\left(P_{n}(I)\right)$. Then:

(1) $M_{n}\left(P_{n}(I)\right) \mathcal{J} M_{n}\left(P_{n}(I)\right)=M_{n}\left(\mathcal{J}^{\prime}\right) \triangleleft M_{n}\left(P_{n}(I)\right)$.

(2) There is a unique $G L_{n}(R)$-action on $M_{n}\left(P_{n}(I) / \mathcal{J}^{\prime}\right)$ such that the natural projection $i: M_{n}\left(P_{n}(I)\right) \rightarrow M_{n}\left(P_{n}(I) / \mathcal{J}^{\prime}\right)$ is $G L_{n}(R)$-equivariant.

(3) $i$ induces a homomorphism

$$
j: M_{n}\left(P_{n}(I)\right)^{G L_{n}(R)} / \mathcal{J} \rightarrow M_{n}\left(P_{n}(I) / \mathcal{J}^{\prime}\right)^{G L_{n}(R)}
$$

which is an isomorphism of $R$-algebras.

Proof. (1) This follows from the basic algebraic fact that every ideal $\mathcal{I}$ in $M_{n}(R)$, for any ring $R$ with 1 , is of the form $M_{n}\left(\mathcal{I}^{\prime}\right)$, where $\mathcal{I}^{\prime}$ is the ideal in $R$ generated by the entries of a generating set of the ideal $\mathcal{I}$.

(2) Let $B \in G L_{n}(R)$, and let $B *$ denote the action of $B$ on $M_{n}\left(P_{n}(I)\right)$. $B$ leaves $M_{n}\left(\mathcal{J}^{\prime}\right)$ invariant. Indeed, any element $C \in M_{n}\left(\mathcal{J}^{\prime}\right)$ is of the form $\sum_{i} M_{i} C_{i} N_{i}$, where $M_{i}, N_{i} \in M_{n}\left(P_{n}(I)\right), C_{i} \in \mathcal{J}$, and therefore

$$
B * C=\sum_{i}\left(B * M_{i}\right)\left(B * C_{i}\right)\left(B * N_{i}\right) \in M_{n}\left(\mathcal{J}^{\prime}\right) .
$$

This implies that the action of $G L_{n}(R)$ on $M_{n}\left(P_{n}(I) / \mathcal{J}^{\prime}\right)$ is well defined. All other statements of (2) are obvious consequences of this fact.

(3) For any rational action of $G L_{n}(R)$ on any $R$-vector space $N$ there exists a linear projection $\nabla: N \rightarrow N^{G L_{n}(R)}$, called the Reynolds operator, with the following properties:

(i) $\nabla(x)=x$ for $x \in N^{G L_{n}(R)}$, and, therefore, $\nabla$ is an epimorphism.

(ii) $\nabla$ is natural with respect to $G L_{n}(R)$-equivariant maps $N \rightarrow N^{\prime}$.

(iii) If $N$ is an algebra, then $\nabla(x y)=x \nabla(y)$ and $\nabla(y x)=\nabla(y) x$ for $x \in N^{G L_{n}(R)}$ and $y \in N$.

For more information about this operator, see $\mathrm{MFK}$ or a more elementary text Fog.

The homomorphism $i$ restricted to $M_{n}\left(P_{n}(I)\right)^{G L_{n}(R)}$ induces a homomorphism

$$
j: M_{n}\left(P_{n}(I)\right)^{G L_{n}(R)} / \mathcal{J} \rightarrow M_{n}\left(P_{n}(I) / \mathcal{J}^{\prime}\right)^{G L_{n}(R)} .
$$

By Property (i) of $\nabla, j$ is an epimorphism. It remains to prove that $j$ is injective.

Choose $i_{0} \in I$. For any monomial $m$ in $P_{n}(I)=R\left[x_{j k}^{i}, i \in I, j, k=1,2, \ldots, n\right]$ we define the degree of $m$ to be the number of appearances of the variables $x_{j k}^{i_{0}}$, $j, k \in\{1,2, \ldots, n\}$, in $m$. This induces a grading on $P_{n}(I)$. We can extend this grading on $M_{n}\left(P_{n}(I)\right)$ as follows. For any matrix $A=\left(a_{j k}\right) \in M_{n}\left(P_{n}(I)\right)$ with a single non-zero entry $a_{s t}, \operatorname{deg}(A)=\operatorname{deg}\left(a_{s t}\right)$. Note that the degree of the matrix $A_{i_{0}}=\left(x_{j k}^{i_{0}}\right) \in M_{n}\left(P_{n}(I)\right)$ considered in Section 2 is 1.

\footnotetext{
${ }^{4}$ Compare also Proposition 9.5 in $[\mathrm{B}-\mathrm{H}]$.
} 
Let $B \in G L_{n}(R)$. By the definition of the $G L_{n}(R)$-action on $M_{n}\left(P_{n}(I)\right)$ and by Lemma 2.2(2),

$$
B\left(\begin{array}{cccc}
B * x_{11}^{i_{0}} & B * x_{12}^{i_{0}} & \ldots & B * x_{1 n}^{i_{0}} \\
\vdots & \vdots & \ldots & \vdots \\
B * x_{n 1}^{i_{0}} & B * x_{n 2}^{i_{0}} & \ldots & B * x_{n n}^{i_{0}}
\end{array}\right) B^{-1}=\left(\begin{array}{cccc}
x_{11}^{i_{0}} & x_{12}^{i_{0}} & \ldots & x_{1 n}^{i_{0}} \\
\vdots & \vdots & \ldots & \vdots \\
x_{n 1}^{i_{0}} & x_{n 2}^{i_{0}} & \ldots & x_{n n}^{i_{0}}
\end{array}\right)
$$

Therefore $B * x_{j k}^{i_{0}}$ is a linear combination of the variables $x_{j^{\prime} k^{\prime}}^{i_{0}}, j^{\prime}, k^{\prime}=1,2, \ldots, n$, and hence the action of $G L_{n}(R)$ preserves the grading of $P_{n}(I)$. For any $M \in$ $M_{n}\left(P_{n}(I)\right), B * M$ is a matrix obtained by applying the action of $B$ to all entries of $M$ and then conjugating the resulting matrix by $B$. Therefore the action of $G L_{n}(R)$ also preserves the grading of $M_{n}\left(P_{n}(I)\right)$. The naturality of the Reynolds operators $\nabla: P_{n}(I) \rightarrow P_{n}(I)^{G L_{n}(R)}$ and $\nabla: M_{n}\left(P_{n}(I)\right) \rightarrow M_{n}\left(P_{n}(I)\right)^{G L_{n}(R)}$ implies that they also preserve the gradings. This fact will be an important element of the proof of Proposition [5.10(3).

We need to show that

$$
\mathcal{J}=M_{n}\left(P_{n}(I)\right) \mathcal{J} M_{n}\left(P_{n}(I)\right) \cap M_{n}\left(P_{n}(I)\right)^{G L_{n}(R)} .
$$

However, it is sufficient to show that

$$
\mathcal{J} \supset M_{n}\left(P_{n}(I)\right) \mathcal{J} M_{n}\left(P_{n}(I)\right) \cap M_{n}\left(P_{n}(I)\right)^{G L_{n}(R)},
$$

since the opposite inclusion is obvious.

Let $c=\sum_{i} a_{i} c_{i} b_{i} \in M_{n}\left(P_{n}(I)\right)^{G L_{n}(R)}$, where $a_{i}, b_{i} \in M_{n}\left(P_{n}(I)\right), c_{i} \in \mathcal{J}$. We will show that $c \in \mathcal{J}$. Since $c$ involves only finitely many variables $x_{j k}^{i}$ and $I$ is infinite, we can choose $i_{0} \in I$ such that $x_{j k}^{i_{0}}, j, k=1,2, \ldots, n$, do not appear in $a_{i}, b_{i}, c_{i}$. Thus $\operatorname{deg} a_{i}=\operatorname{deg} b_{i}=\operatorname{deg} c_{i}=0$.

Consider $\operatorname{Tr}\left(c A_{i_{0}}\right)$. By our assumptions about $c$ and by Lemma 2.2(2), $c A_{i_{0}} \in$ $M_{n}\left(P_{n}(I)\right)^{G L_{n}(R)}$. Proposition 2.3 states that $\operatorname{Tr}: M_{n}\left(P_{n}(I)\right) \rightarrow P_{n}(I)$ is $G L_{n}(R)$ equivariant, and therefore $\operatorname{Tr}\left(c A_{i_{0}}\right) \in M_{n}\left(P_{n}(I)\right)^{G L_{n}(R)}$. Thus

$$
\begin{aligned}
& \operatorname{Tr}\left(c A_{i_{0}}\right)=\operatorname{Tr}\left(\nabla\left(c A_{i_{0}}\right)\right)=\operatorname{Tr}\left(\sum_{i} \nabla\left(a_{i} c_{i} b_{i} A_{i_{0}}\right)\right) \\
& =\operatorname{Tr}\left(\sum_{i} \nabla\left(b_{i} A_{i_{0}} a_{i} c_{i}\right)\right)=\operatorname{Tr}\left(\sum_{i} \nabla\left(b_{i} A_{i_{0}} a_{i}\right) c_{i}\right) .
\end{aligned}
$$

Note that $b_{i} A_{i_{0}} a_{i}$ has degree 1 and, since $\nabla$ preserves the grading, $\nabla\left(b_{i} A_{i_{0}} a_{i}\right)$ is also of degree 1. By The First Fundamental Theorem of Invariant Theory (Theorem 5.1), $M_{n}\left(P_{n}(I)\right)^{G L_{n}(R)}$ is generated by the elements $A_{i}$ and $\operatorname{Tr}(M)$, where $M$ varies over the set of monomials composed of non-negative powers of matrices $A_{i}, i \in I$. By our definition of degree,

$$
\operatorname{deg}\left(A_{i}\right)= \begin{cases}1 & \text { if } i=i_{0} \\ 0 & \text { otherwise }\end{cases}
$$

and

$$
\operatorname{deg}(\operatorname{Tr}(M))=\text { number of appearances of } A_{i_{0}} \text { in } M .
$$


Therefore, $\nabla\left(b_{i} A_{i_{0}} a_{i}\right)$ can be presented as

$$
\sum_{j} p_{i j} A_{i_{0}} q_{i j}+\sum_{k} \operatorname{Tr}\left(s_{i k} A_{i_{0}}\right) t_{i k},
$$

for some elements $p_{i j}, q_{i j}, s_{i k}, t_{i k} \in M_{n}\left(P_{n}(I)\right)^{G L_{n}(R)}$ of degree 0 . Thus

$$
\begin{aligned}
& \operatorname{Tr}\left(c A_{i_{0}}\right)=\operatorname{Tr}\left(\sum_{i} \sum_{j} p_{i j} A_{i_{0}} q_{i j} c_{i}+\sum_{i} \sum_{k} \operatorname{Tr}\left(s_{i k} A_{i_{0}}\right) t_{i k} c_{i}\right) \\
& =\operatorname{Tr}\left(\sum_{i} \sum_{j} p_{i j} A_{i_{0}} q_{i j} c_{i}\right)+\sum_{i} \sum_{k} \operatorname{Tr}\left(s_{i k} A_{i_{0}}\right) \operatorname{Tr}\left(t_{i k} c_{i}\right) \\
& \quad=\operatorname{Tr}\left(\left(\sum_{i} \sum_{j} q_{i j} c_{i} p_{i j}+\sum_{i} \sum_{k} \operatorname{Tr}\left(t_{i k} c_{i}\right) s_{i k}\right) A_{i_{0}}\right) .
\end{aligned}
$$

Therefore

$$
\operatorname{Tr}\left(\left[c-\left(\sum_{i} \sum_{j} q_{i j} c_{i} p_{i j}+\sum_{i} \sum_{k} \operatorname{Tr}\left(t_{i k} c_{i}\right) s_{i k}\right)\right] A_{i_{0}}\right)=0
$$

in $M_{n}\left(P_{n}(I)\right)$. The expression in brackets above has degree 0 . Note that if $d e g d=$ $0, d \in M_{n}\left(P_{n}(I)\right)$, then $\operatorname{Tr}\left(d A_{i_{0}}\right)=0$ if and only if $d=0$. Therefore

$$
c=\sum_{i} \sum_{j} q_{i j} c_{i} p_{i j}+\sum_{i} \sum_{k} \operatorname{Tr}\left(t_{i k} c_{i}\right) s_{i k},
$$

and hence $c \in \mathcal{J}$. This completes the proof of Proposition 5.10 .

Let $\mathcal{J} \triangleleft M_{n}\left(P_{n}(I)\right)^{G L_{n}(R)}$ be the ideal generated by elements $\operatorname{Det}\left(A_{i}\right)-1, i \in I$, and elements $A_{i_{1}} A_{i_{2}} \ldots A_{i_{k}}-1$ corresponding to all defining relations $r_{j}=g_{i_{1}} g_{i_{2}} \ldots g_{i_{k}}$ of $G$. By Lemma $5.9(1)$ and Lemma $2.2(2), \operatorname{Det}\left(A_{i}\right)-1$ and $A_{i_{1}} A_{i_{2}} \ldots A_{i_{k}}-1$ are indeed elements of $M_{n}\left(P_{n}(I)\right)^{G L_{n}(R)}$ and therefore $\mathcal{J}$ is well defined. By Proposition 5.10(1) the ideal $M_{n}\left(P_{n}(I)\right) \mathcal{J} M_{n}\left(P_{n}(I)\right) \triangleleft M_{n}\left(P_{n}(I)\right)$ is equal to $M_{n}\left(\mathcal{J}^{\prime}\right)$, where $\mathcal{J}^{\prime} \triangleleft P_{n}(I)$ is the ideal generated by coefficients of matrices belonging to $\mathcal{J}$. Notice that $\mathcal{J}^{\prime}$ is exactly the kernel of the epimorphism $\eta: P_{n}(I) \rightarrow \operatorname{Rep}_{n}^{R}(G)$ introduced in Section 2. Therefore by Proposition 5.10 the homomorphism

$$
M_{n}(\eta)^{G L_{n}(R)}: M_{n}\left(P_{n}(I)\right)^{G L_{n}(R)} \rightarrow M_{n}\left(R e p_{n}^{R}(G)\right)^{G L_{n}(R)}
$$

considered in diagram (5.5) descends to an isomorphism

$$
j: M_{n}\left(P_{n}(I)\right)^{G L_{n}(R)} / \mathcal{J} \rightarrow M_{n}\left(\operatorname{Rep}_{n}^{R}(G)\right)^{G L_{n}(R)} .
$$

Proposition 5.11. $M_{n}\left(\operatorname{Rep}_{n}^{R}(G)\right)^{G L_{n}(R)}$ is generated by the elements $j_{G, n}\left(g_{i}\right)$ and $\operatorname{Tr}\left(j_{G, n}\left(g_{i_{1}} g_{i_{2}} \ldots g_{i_{k}}\right)\right)$, where $i, i_{1}, i_{2}, \ldots, i_{k} \in I$.

Proof. From the paragraph preceding Proposition 5.11 it follows that $M_{n}(\eta)^{G L_{n}(R)}$ is an epimorphism. By Theorem 5.1, $M_{n}\left(P_{n}(I)\right)^{G L_{n}(R)}$ is generated by the elements $A_{i}$ and $\operatorname{Tr}\left(A_{i_{1}} A_{i_{2}} \ldots A_{i_{k}}\right)$, where $i, i_{1}, i_{2}, \ldots, i_{k} \in I$. The homomorphism $M_{n}(\eta)^{G L_{n}(R)}$ carries these elements to $j_{G, n}\left(g_{i}\right)$ and $\operatorname{Tr}\left(j_{G, n}\left(g_{i_{1}} g_{i_{2}} \ldots g_{i_{k}}\right)\right)$, respectively. 
This proposition and Theorem 3.6 imply that $\Theta$ is an epimorphism. We will show that it is also a monomorphism.

We have shown in Lemma[5.9 that $\operatorname{Det}\left(A_{i}\right)-1 \in \operatorname{Ker} \psi^{\prime} 5$ Moreover, by the definition of $\psi^{\prime}, A_{i_{1}} A_{i_{2}} \ldots A_{i_{k}}-1 \in \operatorname{Ker} \psi^{\prime}$, for any $i_{1}, i_{2}, \ldots, i_{k}$ such that $g_{i_{1}} g_{i_{2}} \ldots g_{i_{k}}=e$ in $G$. Therefore $\mathcal{J} \subset \operatorname{Ker} \psi^{\prime}$, and we can factor $\psi^{\prime}$ to

$$
\psi^{\prime \prime}: M_{n}\left(\operatorname{Rep} p_{n}^{R}(G)\right)^{G L_{n}(R)} \rightarrow \mathbb{A}_{n}\left(X, x_{0}\right),
$$

such that diagram (5.5) commutes and, by Corollary [5.8

- $\psi^{\prime \prime}\left(j_{G, n}\left(g_{i}\right)\right)=E_{g_{i}}$,

- $\psi^{\prime \prime}\left(\operatorname{Tr}\left(j_{G, n}\left(g_{i_{1}} g_{i_{2}} \ldots g_{i_{k}}\right)\right)=E L_{g_{i_{1}} g_{i_{2}} \ldots g_{i_{k}}}\right.$, for any $i_{1}, i_{2}, \ldots, i_{k} \in I$.

Recall that our assumptions about the presentation of $G$ (stated in the paragraph following Theorem [5.3) say that the inverse of any generator of $G$ is also a generator and that every element of $G$ is a product of non-negative powers of generators. Thus, by Proposition $3.5(4), \mathbb{A}_{n}\left(X, x_{0}\right)$ is generated by the elements $E_{g_{i}}$ and $E L_{g_{i_{1}} g_{i_{2}} \ldots g_{i_{k}}}$, for $i, i_{1}, i_{2}, \ldots, i_{k} \in I$. Since $\psi^{\prime \prime} \circ \Theta$ is the identity on the generators of $\mathbb{A}_{n}\left(X, x_{0}\right)$, it also is the identity on $\mathbb{A}_{n}\left(X, x_{0}\right)$. Therefore $\Theta$ is a monomorphism.

In order to complete the proof of Theorem 3.7, we need to show that $\theta$ is also an isomorphism.

Fact 3.4 implies that we have an embedding $\imath_{*}: \mathbb{A}_{n}(X) \rightarrow \mathbb{A}_{n}\left(X, x_{0}\right), \imath_{*}\left(L_{g}\right)=$ $E L_{g}$, for $g \in G$. Therefore we can consider $\mathbb{A}_{n}(X)$ as a subring of $\mathbb{A}_{n}\left(X, x_{0}\right)$. Moreover, by Theorem 3.6, $\theta$ is just the restriction of

$$
\Theta: \mathbb{A}_{n}\left(X, x_{0}\right) \rightarrow M_{n}\left(\operatorname{Rep} p_{n}^{R}(G)\right)^{G L_{n}(R)}
$$

to $\mathbb{A}_{n}(X)$. Therefore $\theta$ is a monomorphism.

In order to show that $\theta$ is an epimorphism we use once again an argument from invariant theory. By the naturality of the Reynolds operators $\nabla: M_{n}\left(\operatorname{Rep}_{n}^{R}(G)\right) \rightarrow$ $M_{n}\left(\operatorname{Rep}_{n}^{R}(G)\right)^{G L_{n}(R)}$ and $\nabla^{\prime}: \operatorname{Rep}_{n}^{R}(G) \rightarrow \operatorname{Rep}_{n}^{R}(G)^{G L_{n}(R)}$, the following diagram commutes:

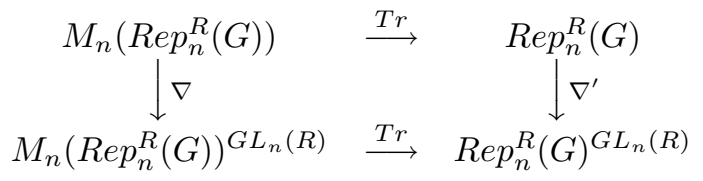

Since $\operatorname{Tr}: M_{n}\left(\operatorname{Rep}_{n}^{R}(G)\right) \rightarrow \operatorname{Rep}_{n}^{R}(G)$ and all Reynolds operators are epimorphic, $\operatorname{Tr}: M_{n}\left(\operatorname{Rep} p_{n}^{R}(G)\right)^{G L_{n}(R)} \rightarrow \operatorname{Re} p_{n}^{R}(G)^{G L_{n}(R)}$ is also epimorphic. But now commutativity of (3.1) implies that $\theta$ is an epimorphism as well.

Therefore we have shown that $\theta$ is an isomorphism. This completes the proof of Theorem 3.7 .

\section{6. $S L_{n}$-CHARACTER VARIETIES}

In this section we present one of the possible applications of Theorem 3.7 to a study of $S L_{n}$-character varieties.

Let $X$ be a path-connected topological space whose fundamental group $G=$ $\pi_{1}(X)$ is finitely generated. Let $K$ be an algebraically closed field of characteristic 0 . Recall that we noticed in Section 2 that the set of all $S L_{n}(K)$-characters of $G$, denoted by $X_{n}(G)$, is an algebraic set whose coordinate ring is $\operatorname{Rep}_{n}^{R}(G)^{G L_{n}(K)} / \sqrt{0}$.

Let $\chi_{g}=\operatorname{Tr}\left(j_{G, n}(g)\right) \in \operatorname{Rep}_{n}^{R}(G)^{G L_{n}(K)} / \sqrt{0}$, for any $g \in G$. It is not difficult to check that $\chi_{g}$, considered as an element of $K\left[X_{n}(G)\right]$, is a function which

${ }^{5}$ Recall that the map $\psi^{\prime}$ was defined in Corollary 5.8 
assigns to a character $\chi$ the value $\chi(g)$. By Proposition 3.5.(3) and Theorem 3.7 $\operatorname{Rep} R(G)^{G L_{n}(K)}$ is generated by the elements $\operatorname{Tr}\left(j_{G, n}(g)\right)$. Therefore the functions $\chi_{g}$ generate $K\left[X_{n}(G)\right]$.

By an $S L_{n}$-trace identity for $G$ we mean a polynomial function in variables $\chi_{g}, g \in G$, which is identically equal to 0 on $X_{n}(G)$. For example,

$$
\chi_{g} \chi_{h}=\chi_{g h}+\chi_{g h^{-1}}
$$

is the famous Fricke-Klein $S L_{2}$-trace identity, valid for any group $G$ and any $g, h \in$ $G$. From the above discussion it follows that the coordinate ring of $X_{n}(G)$ can be considered as the quotient of the ring of polynomials in formal variables $\chi_{g}, g \in G$, by the ideal of all $S L_{n}$-trace identities for $G$. Therefore Theorem 3.7 implies the following corollary.

Corollary 6.1. There is an isomorphism $\Lambda: \mathbb{A}_{n}(X) / \sqrt{0} \rightarrow K\left[X_{n}(G)\right]$ such that $\Lambda\left(L_{g}\right)=\chi_{g}$. Under this isomorphism the identities on graphs in $X$ induced by skein relations correspond to $S L_{n}$-trace identities for $G$.

The above corollary is very useful in the study of trace identities, since it makes it possible to interpret them geometrically. Consider for example the following $S L_{3^{-}}$ trace identity, which holds for any $\gamma_{0}, \gamma_{1}, \gamma_{2}, \gamma_{3} \in G$ and any $\chi \in X_{3}(G)$, where $G$ is an arbitrary group:

$$
\begin{aligned}
& \chi\left(\gamma_{1}\right) \chi\left(\gamma_{2}\right) \chi\left(\gamma_{3}\right)-\chi\left(\gamma_{1}\right) \chi\left(\gamma_{2} \gamma_{3}\right)-\chi\left(\gamma_{2}\right) \chi\left(\gamma_{1} \gamma_{3}\right)-\chi\left(\gamma_{3}\right) \chi\left(\gamma_{1} \gamma_{2}\right) \\
& \quad+\chi\left(\gamma_{1} \gamma_{2} \gamma_{3}\right)+\chi\left(\gamma_{1} \gamma_{3} \gamma_{2}\right)-\chi\left(\gamma_{1} \gamma_{0}\right) \chi\left(\gamma_{2} \gamma_{0}\right) \chi\left(\gamma_{3} \gamma_{0}\right) \\
& \quad+\chi\left(\gamma_{1} \gamma_{0}\right) \chi\left(\gamma_{2} \gamma_{0} \gamma_{3} \gamma_{0}\right)+\chi\left(\gamma_{2} \gamma_{0}\right) \chi\left(\gamma_{1} \gamma_{0} \gamma_{3} \gamma_{0}\right)+\chi\left(\gamma_{3} \gamma_{0}\right) \chi\left(\gamma_{1} \gamma_{0} \gamma_{2} \gamma_{0}\right) \\
& \quad-\chi\left(\gamma_{1} \gamma_{0} \gamma_{2} \gamma_{0} \gamma_{3} \gamma_{0}\right)-\chi\left(\gamma_{1} \gamma_{0} \gamma_{3} \gamma_{0} \gamma_{2} \gamma_{0}\right)=0 .
\end{aligned}
$$

Our theory provides the following interpretation of this identity. Let $x_{0} \in X$ and $G=\pi_{1}\left(X, x_{0}\right)$. Let $\gamma_{0}$ be a path in $X$ representing a non-trivial element of $\pi_{1}\left(X, x_{0}\right)$. We assume that $\gamma_{0}$ goes along a "hole" in $X$ presented in the picture below. Consider the following two, obviously equivalent, graphs $\Gamma$ and $\Gamma^{\prime}$ in $X$ :
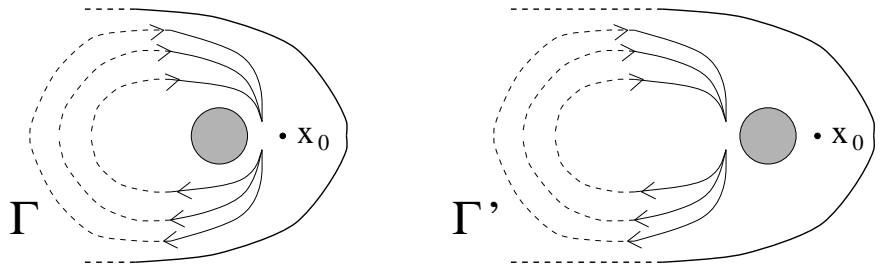

The graph $\Gamma^{\prime}$ is obtained from $\Gamma$ by pulling its vertices along the "hole" in $X$. The obvious resolution of vertices in $\Gamma$ and $\Gamma^{\prime}$ gives an equation involving closed loops in $X$. This equation corresponds to the trace identity (6.1).

There is a large body of literature about $S L_{2}$-character varieties and their applications. However, very little is known about $S L_{n}$-character varieties for $n>2$. The reason for this is that the $S L_{n}$-trace identities, like (6.1), are intractable by classical (algebraic) methods. Since our theory often gives a simple geometric interpretation to complicated trace identities, it can be applied to a more detailed study of character varieties. This idea was already used in $\mathrm{PS}-2]$ and $[\mathrm{PS}-3]$ to study $S L_{n}$-character varieties for $n=2$. A generalization of these results for $n>2$, which is based on our skein method, will appear in future papers. In this work we test our method on the simplest non-trivial example - we study $S L_{3}$-character 
variety of the free group on two generators, $F_{2}=\left\langle g_{1}, g_{2}\right\rangle$. The basic problem is to determine the minimal dimension of the affine space in which $X_{3}\left(F_{2}\right)$ is embedded, or equivalently, the minimal number of generators of $K\left[X_{3}\left(F_{2}\right)\right]$. A result of Procesi (Theorem 3.4(a) [Pro-1]) implies that $K\left[X_{3}\left(F_{2}\right)\right]$ is generated by the elements $\chi_{g_{i_{1}} g_{i_{2}} \ldots g_{i_{j}}}$, where $j \leq 7$ and $i_{1}, i_{2}, \ldots, i_{j} \in\{1,2\}$. A direct calculation shows that, after identifying words in $g_{1}, g_{2}$ which are related by cyclic permutations, we get a set of 57 generators of $K\left[X_{3}\left(F_{2}\right)\right]$. It is difficult to obtain any further reduction of this set in any simple algebraic manner. However, our geometric method allows us to reduce this problem to the study of 3-valent graphs in the twice-punctured disc. By playing with pictures of such graphs one can reduce the number of generators of $K\left[X_{3}\left(F_{2}\right)\right]$ to nine! These are

$$
\chi_{g_{1}}, \chi_{g_{2}}, \chi_{g_{1}^{2}}, \chi_{g_{2}^{2}}, \chi_{g_{1} g_{2}}, \chi_{g_{1}^{2} g_{2}}, \chi_{g_{1} g_{2}^{2}}, \chi_{g_{1}^{2} g_{2}^{2}}, \chi_{g_{1}^{2} g_{2}^{2} g_{1} g_{2}} .
$$

Moreover, it is possible to show that this is the minimal number of generators, and $X_{3}\left(F_{2}\right) \subset K^{9}$ is a solution set of one irreducible polynomial.

\section{REFERENCES}

[BHMV-1] C. Blanchet, N. Habegger, G. Masbaum, P. Vogel, Three-manifold invariants derived from the Kauffman bracket, Topology, 31 (1992), no. 4, 685-699. MR 94a:57010

[BHMV-2] C. Blanchet, N. Habegger, G. Masbaum, P. Vogel, Topological quantum field theories derived from the Kauffman bracket, Topology, 34 (1995), no. 4, 883-927. MR 96i:57015

[B-H] G. W. Brumfiel, H. M. Hilden, $S L(2)$ Representations of Finitely Presented Groups, Contemp. Math. 187 (1995). MR 96g:20004

[B-1] D. Bullock, Estimating a skein module with $S L_{2}(\mathbb{C})$ characters, Proc. Amer. Math. Soc. 125(6), 1997, 1835-1839. MR 97g:57018

[B-2] D. Bullock, Rings of $S L_{2}(\mathbb{C})$-characters and the Kauffman bracket skein module, Comment. Math. Helv. 72, 1997, 521-542. MR 98k:57008

[Co] J. H. Conway, An enumeration of knots and links, and some of their algebraic properties. Computational Problems in Abstract Algebra (Proc. Conf., Oxford, 1967), pp. 329-358, Pergamon, Oxford, 1970. MR 41:2661

[Cv] P. Cvitanović, Group Theory, Part I, Nordita Notes, January 1984.

[Fog] J. Fogarty, Invariant Theory, W. A. Benjamin, Inc. 1969. MR 29:1458

[For] E. Formanek, The Polynomial Identities and Invariants of $n \times n$ Matrices, $C B M S$ Regional Conf. Ser. 78 AMS, 1991. MR 92g:16031

[FYHLMO] P. Freyd, D. Yetter, J. Hoste, W.B.R. Lickorish, K. Millett, A. Ocneanu, A new polynomial invariant of knots and links. Bull. Amer. Math. Soc. (N.S.) 12 (1985), no. 2, 239-246. MR 86e:57007

[H-P] J. Hoste, J.H. Przytycki, A survey of skein modules of 3-manifolds; in Knots 90, Proceedings of the International Conference on Knot Theory and Related Topics, Osaka (Japan), August 15-19, 1990, Editor A. Kawauchi, Walter de Gruyter (1992), 363-379. MR 93m:57018

[Jo] V. F. R. Jones, Hecke algebra representations of braid groups and link polynomials. Ann. of Math. 126 (1987), no. 2, 335-388. MR 89c:46092

[KM] M. Kapovich, J. J. Millson, On representation varieties of Artin groups, projective arrangements and the fundamental groups of smooth complex algebraic varieties, Inst. Hautes Études Sci. Publ. Math. 88, (1998), 5-95 (1999). CMP 2000:07

[Ka] L. H. Kauffman, An invariant of regular isotopy, Trans. Amer. Math. Soc. 318 (1990), no. 2, 417-471. MR 90g:57007

[L1] W. B. R. Lickorish, Three-manifolds and the Temperley-Lieb algebra, Math. Ann. 290 (1991), no. 4, 657-670. MR 92e:57014

[L2] W. B. R. Lickorish, Calculations with the Temperley-Lieb algebra, Comment. Math. Helv. 67 (1992), no. 4, 571-591. MR 94c:57032

[L3] W. B. R. Lickorish, The skein method for three-manifold invariants, J. Knot Theory Ramifications 2 (1993), no. 2, 171-194. MR 94g:57006 
[L-M] A. Lubotzky, A. Magid, Varieties of representations of finitely generated groups, Memoirs of the AMS 336 (1985). MR 87c:20021

[MFK] D. Mumford, J. Fogarty, F. Kirwan Geometric Invariant Theory, Springer-Verlag 1994. MR 95m:14012

[Ny] L. Nyssen, Pseudo-représentations, Mathematische Annalen 306 (1996), 257-283. MR 98a:20013

[Pro-1] C. Procesi, The invariant theory of $n \times n$ matrices, Adv. in Math. 19 (1976), 301-381. MR 54:7512

[Pro-2] C. Procesi, A formal inverse to the Cayley-Hamilton theorem, J. of Alg. 107 (1987), 63-74. MR 88b:16033

[PS-1] J.H. Przytycki, A.S. Sikora, Skein algebra of a group, Proceedings of an International Conference in Knot Theory, Warsaw 1995, Banach Center Publications, 42, Polish Acad. Sci., Warsaw, 1998, pp. 297-306. MR 99e:57019

[PS-2] J.H. Przytycki, A.S. Sikora, On Skein Algebras and $\mathrm{Sl}_{2}(\mathbb{C})$-character varieties, Topology. 39 (2000), 115-148. MR 2000g:57026

[PS-3] J.H. Przytycki, A.S. Sikora, Skein algebras of surfaces, Preprint.

[P-T] J. H. Przytycki, P. Traczyk, Invariants of links of Conway type, Kobe J. Math. 4 (1988), no. 2, 115-139. MR 89h:57006

[Ra] Y. P. Razmyslov, Identities of algebras and their representations, Translations of Mathematical Monographs, 138, AMS 1994. MR 95i:16022

[R-T] N. Reshetikhin, V. G. Turaev, Invariants of 3-manifolds via link polynomials and quantum groups, Invent. Math. 103 (1991), no. 3, 547-597. MR 92b:57024

[Ro-1] J. Roberts, Skein theory and Turaev-Viro invariants, Topology 34 (1995), no. 4, 771787. MR 97g:57014

[Ro-2] J. Roberts, Skeins and mapping class groups, Math. Proc. Cambridge Philos. Soc. 115 (1994), no. 1, 53-77. MR 94m:57035

[Ro] R. Rouquier, Caractérisation des caractèrs et Pseudo-caractères, J. of Algebra 180, no. 0083, (1996) 571-586. MR 97a:20010

[Sa-1] K. Saito, Representation varieties of a finitely generated group into $S L_{2}$ or $G L_{2}$, RIMS Publications 958, Kyoto University (1993).

[Sa-2] K. Saito, Character variety of representations of a finitely generated group in $S L_{2}$, Topology and Teichmüller Spaces (Proc. Taniguchi Sympos., Katinkulta, Finland, 1995; S. Kojima et al., eds.), World Sci. Publ., Singapore, 1996, pp. 253-264. MR 99k:20011

[Si] A.S. Sikora, Spin networks and character varieties, in preparation.

[Ta] R. Taylor, Galois representations associated to Siegel modular forms of low weight, Duke Math. Journal 63 (1991), no. 2, 281-332. MR 92j:11044

[We] H. Weyl, The Classical Groups, Princeton Univ. Press, 1939. MR 1:42c

[Yo] Y. Yokota, Skeins and quantum SU $(N)$ invariants of 3-manifolds. Math. Ann. 307 (1997), no. 1, 109-138. MR 98h:57039

Department of Mathematics, University of Maryland, College Park, Maryland 20742

E-mail address: asikora@math.umd.edu 